Bryn Mawr College

Scholarship, Research, and Creative Work at Bryn Mawr

College

Physics Faculty Research and Scholarship

Physics

2017

\title{
Monitoring a simple hydrolysis process in an organic solid by observing methyl group rotation
}

Peter A. Beckmann

Bryn Mawr College, pbeckman@brynmawr.edu

Joseph M. Bohen

Jamie Ford

William P. Malachowski

Bryn Mawr College

Clelia W.Mallory

See next page for additional authors

Let us know how access to this document benefits you.

Follow this and additional works at: http://repository.brynmawr.edu/physics_pubs

Part of the Chemistry Commons, and the Physics Commons

\section{Custom Citation}

Beckmann P.A., Bohen J.M., Ford J., Malachowski W.P., Mallory C.W., Mallory F.B., McGhie A.R., Rheingold A.L., Sloan G.J., Szewczyk S.T., Wang X., Wheeler K.A. "Monitoring a simple hydrolysis process in an organic solid by observing methyl group rotation." Solid State Nuclear Magnetic Resonance 85 (2017): 1-11.

This paper is posted at Scholarship, Research, and Creative Work at Bryn Mawr College. http://repository.brynmawr.edu/physics_pubs/97

For more information, please contact repository@brynmawr.edu. 


\section{Authors}

Peter A. Beckmann, Joseph M. Bohen, Jamie Ford, William P. Malachowski, Clelia W. Mallory, Frank B.

Mallory, Andrew R. McGhie, Arnold L. Rheingold, Gilbert J. Sloan, Steven T. Szewczyk, Xianlong Wang, and Kraig A. Wheeler 


\section{Monitoring a simple hydrolysis process in an organic solid by observing methyl group rotation}

Peter A. Beckmann, ${ }^{* a}$ Joseph M. Bohen, ${ }^{\mathrm{b}}$ Jamie Ford, ${ }^{\mathrm{c}}$ William P. Malachowski, ${ }^{b}$ Clelia W. Mallory, ${ }^{\mathrm{d}}$ Frank B. Mallory, ${ }^{\mathrm{b}}$ Andrew R. McGhie, ${ }^{\mathrm{e}}$ Arnold L. Rheingold, ${ }^{\mathrm{f}}$ Gilbert J. Sloan, ${ }^{\mathrm{e}}$ Steven T. Szewczyk, ${ }^{\mathrm{g}}$ Xianlong Wang, ${ }^{\mathrm{h}}$ and Kraig A. Wheeler ${ }^{\mathrm{i}}$

a Department of Physics, Bryn Mawr College, 101 North Merion Ave., Bryn Mawr, Pennsylvania 19010-2899, USA.

${ }^{\mathrm{b}}$ Department of Chemistry, Bryn Mawr College, 101 North Merion Ave., Bryn Mawr, Pennsylvania 19010-2899, USA.

${ }^{\mathrm{c}}$ Nanoscale Characterization Facility, Singh Center for Nanotechnology, University of Pennsylvania, 3205 Walnut St., Philadelphia, Pennsylvania 19104-3405, USA.

${ }^{\mathrm{d} D e p a r t m e n t ~ o f ~ C h e m i s t r y, ~ U n i v e r s i t y ~ o f ~ P e n n s y l v a n i a, ~} 231$ South 34 Street, Philadelphia, Pennsylvania 19104-6323, USA.

${ }^{\mathrm{e}}$ Laboratory for Research on the Structure of Matter, University of Pennsylvania, 3231 Walnut St., Philadelphia, Pennsylvania 19104-6202, USA.

${ }_{\mathrm{f}}^{\mathrm{f}}$ Department of Chemistry and Biochemistry, University of California, San Diego, 5128 Urey Hall, 9500 Gilman Dr., La Jolla, California 92093-0358, USA.

${ }^{\mathrm{g}}$ Department of Materials Science and Engineering, School of Engineering and Applied Science, University of Pennsylvania, 3231 Walnut St., Philadelphia, Pennsylvania 191046202, USA.

${ }^{\mathrm{h}}$ Key Laboratory for NeuroInformation of Ministry of Education, School of Life Science and Technology, University of Electronic Science and Technology of China, 4 North Jianshe Rd., $2^{\text {nd }}$ Section, Chengdu, China 610054.

iDepartment of Chemistry, Eastern Illinois University, 600 Lincoln Ave., Charleston, Illinois 69120-3099, USA.

*Author to whom correspondence should be addressed. Electronic address: pbeckman@brynmawr.edu

Solid State Nuclear Magnetic Resonance 2017 85 1-11

keywords: methyl group rotation; molecular solids; single-crystal X-ray diffraction; scanning electron microscopy in organics; electronic structure calculations in molecular clusters; chemical conversion by hydrolysis 
We report a variety of experiments and calculations and their interpretations regarding methyl group $\left(\mathrm{CH}_{3}\right)$ rotation in samples of pure 3-methylglutaric anhydride (1), pure 3methylglutaric acid (2), and samples where the anhydride is slowly absorbing water from the air and converting to the acid $\left[\mathrm{C}_{6} \mathrm{H}_{8} \mathrm{O}_{3}(\mathbf{1})+\mathrm{H}_{2} \mathrm{O} \rightarrow \mathrm{C}_{6} \mathrm{H}_{10} \mathrm{O}_{4}(\mathbf{2})\right]$. The techniques are solid state ${ }^{1} \mathrm{H}$ nuclear magnetic resonance (NMR) spin-lattice relaxation, single-crystal X-ray diffraction, electronic structure calculations in both isolated molecules and in clusters of molecules that mimic the crystal structure, field emission scanning electron microscopy, differential scanning calorimetry, and high resolution ${ }^{1} \mathrm{H}$ NMR spectroscopy. The solid state ${ }^{1} \mathrm{H}$ spin-lattice relaxation experiments allow us to observe the temperature dependence of the parameters that characterize methyl group rotation in both compounds and in mixtures of the two compounds. In the mixtures, both types of methyl groups (that is, molecules of $\mathbf{1}$ and 2) can be observed independently and simultaneously at low temperatures because the solid state ${ }^{1} \mathrm{H}$ spin-lattice relaxation is appropriately described by a double exponential. We have followed the conversion $\mathbf{1} \rightarrow \mathbf{2}$ over periods of two years. The solid state ${ }^{1} \mathrm{H}$ spin-lattice relaxation experiments in pure samples of $\mathbf{1}$ and $\mathbf{2}$ indicate that there is a distribution of NMR activation energies for methyl group rotation in $\mathbf{1}$ but not in $\mathbf{2}$ and we are able to explain this in terms of the particle sizes seen in the field emission scanning electron microscopy images.

\section{Introduction}

Solid state ${ }^{1} \mathrm{H}$ nuclear magnetic resonance (NMR) spin-lattice relaxation experiments [1] can be used to explore the dynamical properties of methyl $\left(\mathrm{CH}_{3}\right)$ groups in solids and provide information concerning interactions at the atomic, molecular, and "several molecule" (clusters of molecules) levels [2]. In these solid samples, methyl group rotation is the only motion occurring on the NMR time scale (approximately $10^{-10}-10^{-5} \mathrm{~s}$ for our experiments). In this paper we report

results using solid state ${ }^{1} \mathrm{H}$ spin-lattice relaxation [1], field emission scanning electron microscopy [3], and high resolution ${ }^{1} \mathrm{H}$ NMR spectroscopy, to examine samples that are composed of two similar molecules (each with a single $\mathrm{CH}_{3}$ group) where, over time, one compound is converting into the other by simple hydrolysis. A sample of 3-methylglutaric anhydride (1; Fig. 1a and c), when exposed to the air, will absorb water and covert to 3-methylglutaric acid (2; Fig. 1b and d) 
$\left[\mathrm{C}_{6} \mathrm{H}_{8} \mathrm{O}_{3}(\mathbf{1})+\mathrm{H}_{2} \mathrm{O} \rightarrow \mathrm{C}_{6} \mathrm{H}_{10} \mathrm{O}_{4}(\mathbf{2})\right]$. For samples of $\mathbf{1}$ left open to the air, this results in very unusual solid state ${ }^{1} \mathrm{H}$ spin-lattice relaxation before the conversion is complete. We have followed this process over two years in a commercial sample and over one year starting with a highly purified sample of $\mathbf{1}$. This hydrolysis process is very common and of no particular interest in and of itself. The novelty in this work is that the sample history, as the anhydride converts to the acid, can be monitored with a technique that exploits a microscopic dynamical process $\left(\mathrm{CH}_{3}\right.$ rotation).

We have also performed solid state ${ }^{1} \mathrm{H}$ spin-lattice relaxation [1], field emission scanning electron microscopy [3], differential scanning calorimetry [4], electronic structure calculations [5], and single crystal X-ray diffraction [6] in pure samples of $\mathbf{1}$ and $\mathbf{2}$ as reference points in order to help interpret the solid state ${ }^{1} \mathrm{H}$ spin-lattice relaxation measurements in the mixtures. By comparing the solid state ${ }^{1} \mathrm{H}$ spin-lattice relaxation results and the field emission scanning electron microscopy images in the pure samples, we find support for a model that relates one of the fitted solid state ${ }^{1} \mathrm{H}$ spin-lattice relaxation parameters to a distribution of NMR activation energies for methyl group rotation [7]. This distribution results from the fact that a non-negligible fraction of methyl groups may have different methyl group rotational barriers than those in the ideal crystal environment because they are near crystal surfaces or crystal imperfections.

The solid state ${ }^{1} \mathrm{H}$ spin-lattice relaxation in all these samples results from $\mathrm{CH}_{3}$ rotation and is modeled in terms of standard NMR relaxation theory [1], with appropriate modifications needed when the relaxation is caused by methyl group rotation [8-10]. The fitted NMR activation energies in the pure samples are in reasonable agreement with the barrier heights for methyl group rotation determined by electronic structure calculations in clusters of molecules based on the Xray structures of the pure crystals, both of which are reported here. The calculations in both isolated molecules and in the clusters allow us, independently of all the experimental techniques, to determine, approximately, the intramolecular and intermolecular contributions to the methyl group rotational barrier [11].

Acid $\rightleftharpoons$ anhydride conversion and acid/anhydride mixtures in a variety of solids have been studied using high resolution NMR spectroscopy [refs. 12, 13, and references therein] but the current study is less complicated than these studies in that the only chemistry involved in the present case is that resulting from a single type of molecule of the acid being formed as a single 
type of molecule of the anhydride absorbs water from the atmosphere. Previous studies have usually involved several forms of the relevant anhydride and/or acid.

Readers not interested in the details of the various experimental techniques and calculations or the details of the rationale behind their interpretations, are invited to proceed directly to the Discussion section.

\section{Experimental Methods}

\subsection{Sample Preparations and Designations}

The compounds (solids at room temperature) 3-methylglutaric anhydride (1) (98\%, mp 315-319 K) and 3-methylglutaric acid (2) (99\%, mp 354-359 K) were purchased from SigmaAldrich. We call these samples, used as is, samples 1A (compound 1) and $2 \mathbf{A}$ (compound 2). A sample of 1 was purified (resulting in sample 1B) by zone refinement [14]. A sample of 2 was purified (resulting in sample 2B) by standard recrystallization techniques. These various samples

were used in the solid state ${ }^{1} \mathrm{H}$ spin-lattice relaxation experiments over various periods of time as outlined in Table 1 and in Section 2.8.

\subsection{A Weight Experiment}

A $7.8 \mathrm{~g}$ sample of 3-methylglutaric anhydride [1, sample 1A (from the supplier)] was placed in a desiccator (at room temperature) with a salt-hydrate $\left(\mathrm{Na}_{2} \mathrm{CO}_{3} \bullet 10 \mathrm{H}_{2} \mathrm{O}\right)$ that maintained a constant relative humidity of approximately $87 \%$ at $293 \mathrm{~K}$. The weight increase of this sample was monitored as a function of time, as 3-methylglutaric anhydride absorbed water and turned into 3-methylglutaric acid.

\subsection{X-ray Diffraction}

Single crystals were taken from purified samples of $\mathbf{1}$ (sample 1B) and $\mathbf{2}$ (sample 2B). They were mounted on a Hampton CryoLoop with Paratone-N oil and data were collected with a Bruker D8 diffractometer using an Ultra rotating-anode generator (Mo) equipped with a high- 
efficiency multi-layer, double-bounce monochromator. All data were collected with $1.0 \mathrm{sec} / 1.0^{\mathrm{O}}$ correlated scans. Structures were solved by direct methods and refined by full-matrix leastsquares analysis on $F^{2}$ using SHELX-2014/7 (G. Sheldrick, Bruker-AXS, Madison, Wisconsin, USA).

\subsection{High Resolution ${ }^{1}$ H Nuclear Magnetic Resonance Spectroscopy}

Using a Bruker $400 \mathrm{MHz}$ NMR Spectrometer, high resolution ${ }^{1} \mathrm{H}$ spectra at $400 \mathrm{MHz}$ were taken of (1) a sample of 1 less than an hour after opening the bottle from the supplier, (2) a sample of 2 less than an hour after opening the bottle from the supplier, and (3) a sample taken from sample $\mathbf{1 A 3}$ (see Table 1), the sample of $\mathbf{1}$ that had been open to the air for two years (via a small hole in the solid state NMR sample tube as discussed in Section 2.8) and used in the solid state ${ }^{1} \mathrm{H}$ spin-lattice relaxation experiments. For all three samples, the solvent was $\mathrm{CD}_{3} \mathrm{OD}$ (deuterated methanol).

\subsection{Differential Scanning Calorimetry}

Differential scanning calorimetry was performed on a TA Instruments Q2000 to characterize thermal transitions in the range of 113 to $373 \mathrm{~K}$. Temperature and enthalpy calibrations were made using indium, and baseline corrections were determined from sapphire standards. Differential scanning calorimetry data were collected at a heating rate of $5 \mathrm{~K} / \mathrm{min}$ under a helium purge. Two samples were used; sample 1B1 (zone refined 1) and sample 2B1 (recrystallized 2). (See Table 1.)

\subsection{Field Emission Scanning Electron Microscopy}

Field emission scanning electron microscopy was performed with several of the same

samples used with the solid state ${ }^{1} \mathrm{H}$ spin-lattice relaxation experiments, using a FEI Quanta 600FEG Field Emission Scanning Electron Microscope. Loose material was randomly sprinkled on carbon tape, thus achieving a variety of orientations for the particles (which may or may not be crystallites). Many crystallites of organic solids are comprised of flakes with one dimension 
much smaller than the other two $[2,7]$ so this procedure potentially allows for a determination of the smallest crystallite dimension because some crystallites will be imbedded in the carbon tape in an edge-on orientation. The electron beam energy was $5 \mathrm{keV}$ and the images were taken under 0.38 Torr air pressure.

\subsection{Electronic Structure Calculations}

Electronic structure calculations were carried out with the Gaussian 09 package of programs [15] running Linux on a PSSC Labs Powerwulf computer. This a Beowulf-class computer with 84 computer cores across 7 nodes.

The isolated-molecule geometries of 3-methylglutaric anhydride (1) and 3-methylglutaric acid (2), taken from the X-ray crystallographic structures of the pure compounds, were subject to a full geometry optimization at the B3LYP/6-31G(d) level and then at the B3LYP/6-311++G(d,p) level. The fully relaxed methyl group rotational potential energy surfaces were calculated at the B3LYP/6-311++G(d,p) level by scanning the methyl group rotational coordinate.

Molecular clusters consisting of 15,21,26, and 31 molecules of 1 and 15 and 21 molecules of $\mathbf{2}$ were constructed from the X-ray crystal structures of the pure compounds by incorporating the nearest neighbors around a molecule with the target methyl group [16].

Examples of clusters in other compounds with methyl groups are shown elsewhere [2,16]. These cluster models simulate the environment of the methyl group in the crystal. Various sizes of the clusters allow us to see the convergent behavior of the methyl group rotational barrier as the cluster size increases. Since the positions of the $\mathrm{H}$ atoms are not accurately determined by the $\mathrm{X}$ ray diffraction experiments $[17,18]$, their positions were optimized at the B3LYP/6-31G(d) level while fixing all the other atoms $(\mathrm{C}$ and $\mathrm{O})$ in the clusters at their $\mathrm{X}$-ray determined positions. The rotational barrier of the methyl group on the central molecule of the cluster was calculated. For this calculation, all the atoms in the central molecule in the clusters of $\mathbf{1}$ and $\mathbf{2}$ were allowed to relax. Also, for this calculation, the ring atoms in all other molecules in the clusters for $\mathbf{1}$ and the five $\mathrm{C}$ atoms on the backbone for clusters of $\mathbf{2}$ were frozen at their $\mathrm{X}$-ray determined positions in the crystal while all the other atoms in these molecules were allowed to relax. The basis set superposition error [5] was not corrected in the clusters since previous studies have shown that the basis set superposition error has little impact on the rotational barriers [2] while the computational 
cost is significant. All the calculations in the clusters were performed at the B3LYP/6-31G(d) level with the Grimme's D3BJ empirical correction for the London dispersion [19, 20].

\subsection{Solid State ${ }^{1} H$ Spin-lattice Relaxation}

Solid state ${ }^{1} \mathrm{H}$ spin-lattice relaxation experiments with 3-methylglutaric anhydride (1) (samples 1A and 1B) and 3-methylglutaric acid (2) (samples 2A and 2B) (see Table 1) were performed at $22.5 \mathrm{MHz}$ (magnetic field $0.529 \mathrm{~T}$ ) at temperatures between 103 and $290 \mathrm{~K}$ in $\mathbf{1}$ and between 130 and $300 \mathrm{~K}$ in 2 using a (perturbation $\pi$ )- $t$-(observe $\pi / 2)-t_{\mathrm{w}}$ pulse sequence. The wait time $t_{\mathrm{w}}$ in the pulse sequence was sufficiently long to allow the magnetization to return to its equilibrium value within $0.1 \%$ [11]. This is particularly important if the relaxation is nonexponential.

Temperature was controlled with a flow of cold nitrogen gas in a home-made variable temperature system and temperature was measured with home-made, silver-soldered, copperconstantan thermocouples imbedded in a part of the sample just outside the NMR coil. As such, the samples were not air tight: the hole in the Teflon tape "seal" through which the thermocouple went was approximately $0.5 \mathrm{~mm}$ in diameter. Absolute temperature was measured to $\pm 2 \mathrm{~K}$ and temperature differences and drifts were monitored to $\pm 0.3 \mathrm{~K}$. The thermocouples used are calibrated to four secondary temperature standards and the calibration is checked every few years.

For 3-methylglutaric anhydride (1), solid state ${ }^{1} \mathrm{H}$ spin-lattice relaxation experiments were performed in sample 1A (directly from the manufacturer) one month after being exposed to the air via a small hole as described above (called sample 1A1), one year after being exposed to the air (called sample 1A2), and two years after being exposed to the air (called sample 1A3).

Experiments were performed in sample 1B (a purified version of 1) very soon after purification (called sample 1B1) and one year later (called sample 1B2). The experimental results in all these samples of $\mathbf{1}$ were different. These various uses of sample $\mathbf{1 A}$ and $\mathbf{1 B}$ are indicated in Table 1.

For 3-methylglutaric acid (1), experiments were performed in sample 2A (directly from the manufacturer) soon after being opened (called sample 2A1) and one year later after being exposed to the air via the small hole for the year (called sample 2A2). Finally, experiments were performed once in a purified sample 2B (also called sample 2B1). All the results in the various samples of 3-methylglutaric acid (2) are the same, indicating that the compound is very stable. 
The NMR relaxation experiments also do not distinguish between a 99\% sample from the supplier (sample 2A) and a much purer sample (sample 2B). These various samples of $\mathbf{2}$ are listed in Table 1.

\section{A Review of the ${ }^{1} \mathbf{H}$ Spin-lattice Relaxation Model}

The ${ }^{1} \mathrm{H}$ spin-lattice relaxation results from the modulation of ${ }^{1} \mathrm{H}-{ }^{1} \mathrm{H}$ spin-spin (dipolar) interactions by methyl group $\left(\mathrm{CH}_{3}\right)$ rotation $[11,21]$. When methyl group rotation is responsible for the relaxation, the relaxation is nonexponential near the maximum in the relaxation rate and at higher temperatures [2, 8-10, 22-28]. The recovery of the perturbed magnetization $M(0)$ at these higher temperatures can be fitted by a stretched exponential; $M(t)=[M(\infty)+\{M(0)-$

$M(\infty)\}\left\{\exp \left(-R^{*} t\right)^{\beta}\right\}$, where $R^{*}$ is the characteristic relaxation rate and $\beta$ is the stretching parameter $[11,22,29-35]$. If the relaxation is exponential to within experimental uncertainty, $\beta=$ 1 and $R^{*}$ is labeled $R$. This occurs in pure compounds like $\mathbf{1}$ and $\mathbf{2}$ at temperatures below the maximum in the relaxation rate $[2,8-10,22-28] . R^{*}$ and $\beta$ are not amenable to interpretation in any closed-form model and we use $\beta$ solely as an indicator of the degree of nonexponentiality. When the relaxation is nonexponential, within experimental uncertainty, the parameter that can be modeled, as described below, is $R_{\mathrm{S}}$, the initial decay of the relaxing magnetization [9]. The procedure for determining $R_{\mathrm{S}}$ from $M(t)$ versus $t$ at higher temperatures is indicated elsewhere [22] and a specific model for the entire relaxation curve $M(t)$ is irrelevant. At lower temperatures where the relaxation is exponential, $R_{\mathrm{S}}=R^{*}=R$ and the entire $M(t)$ versus $t$ is fitted. There are three adjustable parameters for exponential relaxation $[M(0), M(\infty)$, and $R]$ and four adjustable parameters for stretched exponential relaxation $\left[M(0), M(\infty), \beta\right.$, and $\left.R^{*}\right]$. Ideally, $M(0)=-M(\infty)$ for a perturbation $\pi$ pulse but the $\pi$ pulse is not perfect and if $M(0)$ is not taken as an independent adjustable parameter, significant systematic errors in the other parameters can result.

The previous paragraph refers to ${ }^{1} \mathrm{H}$ spin-lattice relaxation in the pure compounds $\mathbf{1}$ and $\mathbf{2}$. In samples where both compounds are present, the decay of a perturbed ${ }^{1} \mathrm{H}$ magnetization at lower temperatures is fitted with a double exponential $M(t)=\left[M_{1}(\infty)+\left\{M_{1}(0)-\right.\right.$ $\left.\left.M_{1}(\infty)\right\}\left\{\exp \left(-R_{1} t\right)\right\}\right]+\left[M_{2}(\infty)+\left\{M_{2}(0)-M_{2}(\infty)\right\}\left\{\exp \left(-R_{2} t\right)\right\}\right]$. The subscripts 1 and 2 refer to the two components of the relaxing magnetization which are identified with compounds $\mathbf{1}$ and $\mathbf{2}$. 
At these lower temperatures, the relaxation would be exponential if only a single component (i.e. a single compound) were present. A double exponential involves five adjustable parameters which can be taken to be $M(0), M_{1}(\infty), M_{2}(\infty), R_{1}$, and $R_{2}$ where $M(0)=M_{1}(0)+M_{2}(0)$ but not both $M_{1}(0)$ and $M_{2}(0)$ are independent. It is convenient in reporting results to replace $M_{1}(\infty)$ and $M_{2}(\infty)$ with the fractional equilibrium magnetizations $\phi_{\mathrm{k}}(\mathrm{k}=1,2)$ where $\phi_{\mathrm{k}}=M_{\mathrm{k}}(\infty) /\left[M_{1}(\infty)+\right.$ $\left.M_{2}(\infty)\right]$. The parameters $\phi_{1}$ and $\phi_{2}$ will be interpreted as, approximately, the fractions of the sample that are compound $\mathbf{1}$ and $\mathbf{2}$. This interpretation neglects the fact that the nuclear magnetization for 3-methylglutaric anhydride arises from seven ${ }^{1} \mathrm{H}$ spins whereas the nuclear magnetization for 3-methylglutaric acid arises from nine ${ }^{1} \mathrm{H}$ spins. We will quote $\phi_{1}$ and $\phi_{2}$ to $\pm 10 \%$ so we neglect this simplification. It is important to note that when both compounds are present, a four-parameter stretched exponential does not fit $M(t)$ at low temperatures.

Determining a mathematical model for $M(t)$ with the least number of adjustable parameters is important input into modeling the relationship between $\mathrm{CH}_{3}$ rotation and the structure of the sample on the mesoscopic scale, as discussed in the Results section.

The initial relaxation rate $R_{\mathrm{S}}$ at higher temperatures in all samples, the single relaxation rate $R$ at lower temperatures in the pure samples, and the two relaxation rates $R_{1}$ and $R_{2}$ at lower temperatures in the samples where both compounds $\mathbf{1}$ and $\mathbf{2}$ are present, are all modeled by $R=$ $(n / N)(1+\mathrm{z}) A_{\mathrm{intraCH} 3}[J(\omega, \tau)+4 J(2 \omega, \tau)]$ with $J(\omega, \tau)=(2 / \omega)\left[\sin \{\varepsilon \arctan (\omega, \tau)] /\left\{\left(1+\omega^{2} \tau^{2}\right)^{\varepsilon / 2}\right\}\right][7$, 36, 37]. As is verified by experiment, at lower temperatures in samples where both compounds 1 and 2 are present, $R_{1}$ and $R_{2}$ in the double exponential for $M(t)$ are the same as the corresponding $R$ values in the pure compounds. We note that we do not need to consider the quantum mechanical tunneling of methyl groups at the high temperatures encountered here [21, 38-45]. In these expressions for $R$ and $J(\omega, \tau), \tau=\tau_{\infty}\left[\exp \left\{E_{\mathrm{NMR}} / k T\right\}\right], \tau_{\infty}=x(2 \pi / 3)\left(2 I / E_{\mathrm{NMR}}\right)^{1 / 2}[38,46-48]$, and $A_{\text {intraCH3 }}=(9 / 40)\left[\mu_{\mathrm{O}} /\left(4 \pi^{2}\right)\right]^{2}\left(\hbar \gamma^{2} / r^{3}\right)^{2}$ where $\mu_{\mathrm{O}}$ is the magnetic constant and $\gamma$ is the proton magnetogyric ratio. In fitting the temperature dependence of the various relaxation rates, the adjustable parameters are $E_{\mathrm{NMR}}, \varepsilon, x$, and $z . E_{\mathrm{NMR}}$ is an NMR activation energy that is closely related to, but, in fact, is different from (and probably slightly smaller than), the barrier for $\mathrm{CH}_{3}$ rotation in compounds like those investigated here [49-51]. The parameter $x$ in $\tau_{\infty}=$ $x(2 \pi / 3)\left(2 I / E_{\mathrm{NMR}}\right)^{1 / 2}$ accounts for the departure from $\tau_{\infty}=(2 \pi / 3)\left(2 I / E_{\mathrm{NMR}}\right)^{1 / 2}$, the latter being a very crude model for the preexponential factor [38, 46-48]. The parameters $\varepsilon$ and $z$ are discussed 
below. $J(\omega, \tau)$ is the spectral density (discussed further in the next paragraph), $\omega /(2 \pi)=22.5 \mathrm{MHz}$ is the NMR frequency, $\tau$ is the mean time between methyl group hops in a semiclassical methyl group hopping process, $I$ is the moment of inertia of a methyl group, $n=3$ is the number of ${ }^{1} \mathrm{H}$ spins in a methyl group, and $N(=7$ in $\mathbf{1}$ and 9 in 2$)$ is the number of ${ }^{1} \mathrm{H}$ spins in the asymmetric unit in the crystal (which is a single molecule for both $\mathbf{1}$ and $\mathbf{2}$ ). The above expression for the various relaxation rates assumes that ${ }^{1} \mathrm{H}-{ }^{1} \mathrm{H}$ spin diffusion maintains a common spin temperature for the sample throughout the spin-lattice relaxation process. When both compounds are present in the same sample and a double exponential function is used for $M(t)$, rapid spin diffusion occurs within each of the two magnetizations, but not between them.

From the perspective of interpreting the relaxation rate data in the pure compounds $\mathbf{1}$ and 2, the Davidson-Cole spectral density $J(\omega, \tau)=(2 / \omega)\left[\sin \{\varepsilon \arctan (\omega, \tau)] /\left\{\left(1+\omega^{2} \tau^{2}\right)^{\varepsilon / 2}\right\}\right]$ predicts $[2,7,37]$ (i) an NMR frequency-independent linear $\ln R$ versus $T^{1}$ at high temperatures $(\omega \tau<<1)$, (ii) a frequency-dependent linear $\ln R$ versus $T^{1}$ at low temperatures ( $\left.\omega \tau \gg>1\right)$, and (iii) different (magnitudes of) slopes in $\Delta \ln R / \Delta T^{1}$ at high and low temperatures. The parameter $\varepsilon$ is just the ratio of these slopes. No other (closed form) spectral density has these properties and these properties are observed in many experiments. The Davidson-Cole spectral density has only one additional parameter $(\varepsilon)$ from those found in the Poisson (unique $\tau$ ) spectral density $J(\omega, \tau)=2 \tau /(1$ $\left.+\omega^{2} \tau^{2}\right) . J(\omega, \tau)=(2 / \omega)\left[\sin \{\varepsilon \arctan (\omega, \tau)] /\left\{\left(1+\omega^{2} \tau^{2}\right)^{\varepsilon / 2}\right\}\right]$ can be expressed, in closed form, as a distribution of values of $\tau$ in the functions $J(\omega, \tau)=2 \tau /\left(1+\omega^{2} \tau^{2}\right)$ [37]. The resulting distribution of $\tau$ values [7,37] is characterized solely by $\varepsilon$. Via $\tau=\tau_{\infty}\left[\exp \left\{E_{\mathrm{NMR}} / k T\right\}\right]$, the distribution in $\tau$ values can be cast into a distribution of $E_{\mathrm{NMR}}$ values. Plots are provided in refs. 7 and 37 . In the limit $\varepsilon \rightarrow 1, J(\omega, \tau)=(2 / \omega)\left[\sin \{\varepsilon \arctan (\omega, \tau)] /\left\{\left(1+\omega^{2} \tau^{2}\right)^{\varepsilon / 2}\right\}\right] \rightarrow J(\omega, \tau)=2 \tau /\left(1+\omega^{2} \tau^{2}\right)$ and the distribution of $\tau$ values (or $E_{\mathrm{NMR}}$ values) becomes a Dirac $\delta$-function.

The strength of the ${ }^{1} \mathrm{H}-{ }^{1} \mathrm{H}$ spin-spin interactions are characterized by $A_{\text {intracH3 }}+A_{\text {other }}=$ $A_{\text {intraCH3 }}(1+z)$ where $\mathrm{z}=A_{\text {other }} / A_{\text {intrach3}}$. This is a convenient phenomenological parameterization because $A_{\text {intraCH} 3}$, which accounts for the modulation, by methyl group rotation, of the six pairwise intraCH $\mathrm{H}_{3}$ spin-spin interactions among the three ${ }^{1} \mathrm{H}$ spins in a $\mathrm{CH}_{3}$ group, can be computed with reasonably high precision. This term in $A_{\text {intraCH3 }}$ dominates the relaxation rate. Computing $A_{\text {other, }}$, which accounts for the modulation, by methyl group rotation, of interactions between $\mathrm{CH}_{3}{ }^{1} \mathrm{H}$ 
spins and ${ }^{1} \mathrm{H}$ spins not in $\mathrm{CH}_{3}$ groups (or at least not in the same $\mathrm{CH}_{3}$ group), would be a very complicated and essentially intractable task $[52,53]$. The phenomenological fitting parameter $z$ can be determined by experiment. If $z$ is not significantly less than 1 , this approach is not justified. The $\mathrm{H}-\mathrm{H}$ distances between the three $\mathrm{H}$ atoms in a $\mathrm{CH}_{3}$ group is taken to be $r_{\text {intraCH3 }}=$ $0.170 \mathrm{~nm}$. This is determined by the electronic structure calculations in the clusters presented here since X-ray diffraction experiments give $\mathrm{C}-\mathrm{H}$ bond lengths that are approximately $0.01 \mathrm{~nm}$ too short $[17,18]$, resulting in H-H distances in a methyl group that are also too short (typically $0.16 \mathrm{~nm})$. Since $A_{\text {intraCH3 }} \propto r^{-6}$, a $1 / 16=6.3 \%$ error in $r$ results in a $38 \%$ error in $A_{\text {intraCH3}}$.

\section{Results and Analyses}

\subsection{A Weight Experiment}

A $7.8 \pm 0.1 \mathrm{~g}$ sample of 3-methylglutaric anhydride [1, sample 1A (from the supplier)] was placed in an environment that maintained a constant relative humidity of approximately $87 \%$. The weight as a function of time $W(t)$ is presented in Fig. 2 and is well fitted by $W(t)=W(\infty)+$ $[W(0)-W(\infty)]\left[\exp \left(-t / T_{\mathrm{E}}\right)\right]$ where $T_{\mathrm{E}}=24 \pm 2$ days. This value of $T_{\mathrm{E}}$ (an exposure time constant) has no fundamental importance and will depend on humidity, temperature, and the ratio of surface area to volume of the sample. The latter was large and the humidity was high, so the importance of the value of the time constant is that even in these "extreme" conditions (compared with the samples used in the solid state ${ }^{1} \mathrm{H}$ spin-lattice relaxation experiments), it is very much longer than a few hours or even a few days. This makes this result consistent with the solid state ${ }^{1} \mathrm{H}$ spinlattice relaxation experiments presented in Section 4.7. If the starting sample was all $\mathbf{1}$ and the final sample was all 2 , the theoretical value of $W(\infty) / W(0)$ would be $(145 \mathrm{amu}) /(127 \mathrm{amu})=1.14$, independently of any fitting function. Using the first and last data points in Fig. 2 we obtain $W_{\text {last }} / W_{\text {first }}=(8.7 \pm 0.1 \mathrm{~g}) /(7.8 \pm 0.1 \mathrm{~g})=1.12 \pm 0.03$, again, independently of any fitting function If this ratio were greater than the theoretical value (within experimental uncertainty) then the result would be inconsistent with $\mathbf{1} \rightarrow \mathbf{2}$ via simple hydrolysis. Since this experiment was done with the sample from the supplier (sample 1A), it shows that the $98 \%$ purity quoted was reasonable. 


\subsection{X-ray Diffraction}

The X-ray diffraction data are summarized in Table 2. The structure of the molecules $\mathbf{1}$ and $\mathbf{2}$ in the crystal are shown in Fig. 1 and the crystal structures of $\mathbf{1}$ and $\mathbf{2}$ are shown in Fig. 3. The structures have been deposited with the Cambridge Structural Database and the deposit numbers are 1477660 (1) and 1477661 (2). The asymmetric unit in both crystals is a single molecule $(Z=1)$. Since there is one methyl group per molecule this means that all methyl groups in the bulk crystal are dynamically equivalent. This is important in interpreting the solid state ${ }^{1} \mathrm{H}$ spin-lattice relaxation experiments presented in Section 4.7. It is likely that there will be Hbonding in 2 (see the very center of the crystal structure in Fig. 3 b). If so, it has no consequences for the dynamical models being presented here.

\subsection{High Resolution ${ }^{1}$ H Nuclear Magnetic Resonance Spectroscopy}

High resolution ${ }^{1} \mathrm{H}$ NMR spectra at $400 \mathrm{MHz}$ indicate that the samples of $\mathbf{1}$ and $\mathbf{2}$ from the supplier were consistent with the quoted purities ( $98 \%$ for $\mathbf{1}$ and $99 \%$ for $\mathbf{2}$ ). The spectra with sample $\mathbf{1 A 3}$ (compound $\mathbf{1}$ open to the air through a small hole in the solid state NMR tube for two years) indicate that the sample was approximately $5 \% 1$ and $95 \% 2$. As presented below, this is an important confirmation of the interpretation of the solid state ${ }^{1} \mathrm{H}$ spin-lattice relaxation experiments presented in Section 4.7.

\subsection{Differential Scanning Calorimetry}

Neither sample 1B1 [zone refined 3-methylglutaric anhydride (1)] or sample 2B1 [recrystallized 3-methylglutaric acid (2)] showed any solid-solid phase transitions between 123 and $293 \mathrm{~K}$. This means that the crystal structures for $\mathbf{1}$ and $\mathbf{2}$ determined at $100 \mathrm{~K}$ by the singlecrystal X-ray diffraction experiments are valid for the entire temperature range. Sample 1B1 showed an extrapolated melting point at $317.3 \mathrm{~K}$ with a heat of melting of $124.0 \mathrm{~J} / \mathrm{g}$ and sample 2B1 showed an extrapolated melting point at $356.8 \mathrm{~K}$ with a heat of melting of $180 \mathrm{~J} / \mathrm{g}$. These 
results indicate that these samples are very pure. In addition, we note that the melting points are within the melting point ranges of the samples provided by the supplier (samples $1 \mathbf{A}$ and $\mathbf{2 A}$ ).

\subsection{Field Emission Scanning Electron Microscopy}

Figs. 4 and 5 show field emission scanning electron microscopy images of (small parts of) the same samples of pure $\mathbf{1}$ (sample 1B1) and $\mathbf{2}$ (sample 2B1) used in the solid state ${ }^{1} \mathrm{H}$ spinlattice relaxation experiments.

For pure 3-methylglutaric anhydride (1, sample 1B1), parts b, c, and d of Fig. 4 show different parts of the field of view in part a, all at ten times the resolution of part a. These images indicate a great variety of morphologies and particle sizes with few of the many smallest structures even being indicative of single crystals at the hundreds of nanometers scale. The images for 3-methylglutaric acid (2, sample 2B1) (Fig. 5) show large, smooth particles that are clearly single crystals, very few of which have a smallest dimension smaller than approximately 1 $\mu \mathrm{m}$. These observations are important in interpreting the solid state ${ }^{1} \mathrm{H}$ spin-lattice relaxation results (Section 4.7) in the pure compounds.

For the images of 3-methylglutaric anhydride (1), at the resolution of Fig. 4 b, c, and d, there was some local melting caused by the electron beam. There will have been some water in the chamber (in the 0.38 Torr of air), and there may have been some conversion from $\mathbf{1}$ to 2 during this melting. This is pure conjecture but the large smooth crystals (approximately $10 \mu \mathrm{m}$ $\mathrm{X} 10 \mu \mathrm{m} \mathrm{X} \mathrm{100s} \mathrm{nm)} \mathrm{in} \mathrm{the} \mathrm{image} \mathrm{of} \mathbf{1}$ in Fig. $4 \mathrm{c}$ look remarkably similar to the images of $\mathbf{2}$ in Fig. 5 b. There was no melting for compound 2 .

Field emission scanning electron microscopy images of three other samples of 3methylglutaric anhydride (1) are not shown. A sample directly from the supplier's bottle (that had, by this time, been opened many times) had parts that looked like the step structures in Fig. 5 a and other parts that appeared structureless like Fig. 4 a. Images of samples with histories comparable with samples $\mathbf{1 A 1}$ and $\mathbf{1 A 2}$ (Table 1) show little or no structure. We can conclude that samples of $\mathbf{1}$ where some (samples $\mathbf{1 A 1}$ and $\mathbf{1 A 2}$ ) or most (sample 1A3) of the sample had converted to 2, (see Table 1) were very different in appearance at the sub $\mu \mathrm{m}$ scale than the very stable recrystallized sample of $\mathbf{2}$ (like sample $\mathbf{2 B 1}$ ). 


\subsection{Electronic Structure Calculations}

The calculated ground state structures of isolated molecules of $\mathbf{1}$ and $\mathbf{2}$ are as shown in Fig. 1, meaning that at the resolution shown, the structures of the molecules are the same for the isolated molecules as they are in the crystal. The $\mathrm{C}-\mathrm{C}$ and $\mathrm{C}-\mathrm{O}$ bond lengths determined by single crystal X-ray diffraction are accurately reproduced by the geometry optimization of the isolatedmolecule calculations at the B3LYP/6-311++G(d,p) level. The methyl group adopts a staggered conformation with respect to the $\mathrm{C}-\mathrm{H}$ bond on the $\mathrm{C}$ atom directly connected to the methyl group in $\mathbf{1}$ and 2. The potential energy surface for methyl group rotation has three-fold symmetry in $\mathbf{1}$ and 2. The barrier height for methyl group rotation, defined as the energy difference between the transition state (the eclipsed conformation) and the ground state (the staggered conformation), is calculated to be $13.1 \mathrm{~kJ}^{-1}$ in an isolated molecule of 1 and $13.8 \mathrm{~kJ}^{-1}$ mole ${ }^{-1}$ in an isolated molecule of 2 .

Four clusters $(15,21,26$ and 31 molecules) of 1 were built to simulate the methyl group environment in the crystal structure. The barrier for the rotation of a methyl group on a molecule in the center of the cluster is calculated in the different size clusters. The potential energy surfaces in the cluster calculations have a similar shape to that found in the isolated molecule: the staggered conformation is the ground state and the eclipsed conformation is the transition state. The barrier heights in $\mathbf{1}$ are $18.2 \mathrm{~kJ}$ mole ${ }^{-1}$ in the 15-molecule cluster (i.e., for a methyl group on a molecule at the center of the cluster), $16.5 \mathrm{~kJ} \mathrm{~mole}^{-1}$ in the 21 -molecule cluster, $15.7 \mathrm{~kJ} / \mathrm{mol}$ in the 26-molecule cluster, and $16.0 \mathrm{~kJ}$ mole $^{-1}$ in the 31 -molecule cluster. From these values, we may conclude that intermolecular interactions involving molecules that are far away from the target molecule do not contribute significantly to the methyl group rotational barrier due to the angular isotropy of the interactions but that they have an indirect impact by affecting the structural relaxation of the central molecule as the methyl group on that molecule is rotated. We will call the computed methyl group barrier in a crystal of $116 \mathrm{~kJ} \mathrm{~mole}^{-1}$. Based on these results we performed calculations in 15- and 21-molecule clusters of 2 . The barriers were computed to be $19.9 \mathrm{~kJ}$ mole $^{-1}$ and $20.0 \mathrm{~kJ}$ mole ${ }^{-1}$ and we will call the computed methyl group barrier in a crystal of $220 \mathrm{~kJ} \mathrm{~mole}^{-1}$. These barriers are discussed further in the Discussion Section (Section 5). 


\subsection{Solid State ${ }^{I} H$ Spin-lattice Relaxation}

The temperature dependence of the parameters that characterize the solid state ${ }^{1} \mathrm{H}$ spinlattice relaxation is presented in Figs. 6, 7, 8, and 9. We first fit the temperature dependence of the appropriate relaxation rates [the initial (short-time) relaxation rate $R_{\mathrm{S}}$ at higher temperatures $(\omega \tau<1)$ and in the vicinity of the relaxation rate maximum $(\omega \tau \sim 1)$ and the exponential relaxation rates $R\left(=R_{\mathrm{S}}\right)$ at lower temperatures $\left.(\omega \tau>1)\right]$ in the pure samples of $\mathbf{1}$ (sample 1B1) and 2 (sample 2B1) presented in Fig. 6. Although $R_{\mathrm{S}}$ was determined at all temperatures for consistency (and plotted in Fig. 6), the relaxation was exponential $\left(\beta>0.95, R_{\mathrm{S}}=R^{*}=R\right.$, the unique relaxation rate in a single exponential decay) within experimental uncertainty at lower temperatures. (Plots of both $R_{\mathrm{S}}$ and $R^{*}$ for two methoxyphenanthrenes can be seen in ref. 2.) For pure 3-methylglutaric anhydride (1), $E_{\mathrm{NMR}}=12.4 \pm 1.3 \mathrm{~kJ} \mathrm{~mole}^{-1}, z=0.15 \pm 0.08, \varepsilon=0.80 \pm 0.07$, and $x=3.5 \pm 1$.7. For pure 3-methylglutaric acid (2), $E_{\mathrm{NMR}}=16.0 \pm 1.6 \mathrm{~kJ} \mathrm{~mole}^{-1}, z=0.05 \pm$ $0.02, \varepsilon \equiv 1$, and $x=1.6 \pm 0.8$. The values of $E_{\mathrm{NMR}}, x$, and $z$ indicate that the motion responsible for the ${ }^{1} \mathrm{H}$ spin-lattice relaxation is $\mathrm{CH}_{3}$ rotation. The fits indicate that the modulation of $\mathrm{CH}_{3}-$ non- $\mathrm{CH}_{3}$ (both intramolecular and intermolecular) and interCH${ }_{3}{ }^{1} \mathrm{H}-{ }^{1} \mathrm{H}$ intermolecular spin-spin interactions are contributing (as determined by $z$ ) $7-23 \%$ of the relaxation in 1 and $3-7 \%$ of the relaxation in 2. The main reason for the smallness of $z$, despite the large number of "other" (i.e., the non intraCH $\mathrm{H}_{3}{ }^{1} \mathrm{H}-{ }^{1} \mathrm{H}$ spin-spin interactions involved, is that the contribution of each ${ }^{1} \mathrm{H}-{ }^{1} \mathrm{H}$ interaction is proportional to $r^{-6}$ (where $r$ is the appropriate $\mathrm{H}-\mathrm{H}$ distance) and an additional angular factor that is less than unity [54].

There is a very significant difference between the temperature dependence of the relaxation rates in the pure compounds of $\mathbf{1}$ and $\mathbf{2}$ (Fig. 6). In pure 3-methylglutaric acid (2), $\varepsilon$ is identically 1. It was not taken as an adjustable parameter. This means that the spectral density is given by $J(\omega, \tau)=2 \tau /\left(1+\omega^{2} \tau^{2}\right)$ and not by $J(\omega, \tau)=(2 / \omega)\left[\sin \{\varepsilon \arctan (\omega, \tau)] /\left\{\left(1+\omega^{2} \tau^{2}\right)^{\varepsilon / 2}\right\}\right]$ and that methyl group rotation in $\mathbf{2}$ is modeled by a "text book" hopping model characterized by Poisson statistics with a unique mean time $\tau$ between "events" (hops). [The spectral density 
$J(\omega, \tau)=2 \tau /\left(1+\omega^{2} \tau^{2}\right)$ is the Fourier Transform of the correlation function $g(t)=\exp (-|t| / \tau)$ which can be thought of as an (unormalized) Poisson probability for no hops in the time $t$ given that a hop occurred at time $t=0$.] This interpretation is unambiguous. There are not many examples (for methyl group rotation being responsible for the relaxation) of $\varepsilon=1[2,7,22]$.

For pure 3-methylglutaric anhydride (1), $\varepsilon=0.80 \pm 0.07$. This means that the spectral density $J(\omega, \tau)=(2 / \omega)\left[\sin \{\varepsilon \arctan (\omega, \tau)] /\left\{\left(1+\omega^{2} \tau^{2}\right)^{\varepsilon / 2}\right\}\right]$ is required. This, in turn, means that there is a distribution of correlation times $\tau$ [7], or via $\tau=\tau_{\infty}\left[\exp \left\{E_{\mathrm{NMR}} / k T\right\}\right]$, a distribution of NMR activation energies $E_{\mathrm{NMR}}$. One physical model for the origin of this phenomenon is that $\mathrm{CH}_{3}$ groups near a crystallite surface [or near some other crystal imperfection (dislocations, surface steps, vacancies, impurities, etc.)] see a different barrier than $\mathrm{CH}_{3}$ groups in the 'ideal' crystal environment and as such both the intermolecular contribution to the rotational barrier and the effect the environment has on the intramolecular contribution to the barrier may be different. These kinds of surface effects are well established for atomic solids [55] though that may or may not be relevant for these van der Waals molecular solids. Ref. 7 provides plots of this distribution of $E_{\mathrm{NMR}}$ values for $\varepsilon=0.79$ which can serve as the case of $\varepsilon=0.80$ found here for $\mathbf{1}$. The field emission scanning electron microscopy images of the sample of 1 (Fig. 4) used in the solid state ${ }^{1} \mathrm{H}$ spin-lattice relaxation rate experiments support this interpretation. There are many very small "particles" that do not have a crystalline appearance even at the few hundreds of nanometers scale. At the same time $\varepsilon \equiv 1$ for $\mathbf{2}$ correlates well with the field emission scanning electron microscopy images (Fig. 5) of the sample of $\mathbf{2}$ used in the relaxation experiments. The crystals are large and smooth (having a smallest dimension of more than $1 \mu \mathrm{m}$ ) and will have a negligible fraction of molecules near a surface or other crystal imperfection. As such, there is a single "type" of methyl group, $E_{\mathrm{NMR}}$ is unique, and a Poisson spectral density $J(\omega, \tau)=2 \tau /\left(1+\omega^{2} \tau^{2}\right)$ is appropriate. To put this into perspective, we present an order of magnitude calculation of the fraction of molecules on or near a surface of a crystallite or at some other dislocation. We take the linear dimension of a molecule as $\ell=1 \mathrm{~nm}$ and consider a crystallite that is infinite in two dimensions and whose thickness is $d$. Assume the first five "planes" of molecules into the surface have methyl group rotational barriers that are different from the "perfect crystal" barrier. In addition, there will be dislocations within crystallites and they will mimic crystallite surfaces. With these assumptions and approximations, the fraction of methyl groups that will have an NMR 
activation energy different from the ideal crystal value will be $10 \ell / d$, the factor of $2 \times 5=10$ accounting for both surfaces. If we take $d=100 \mathrm{~nm}$, then $10 \%$ of the molecules would have an NMR activation energy different from the ideal crystal value. We conclude that the origin of $\varepsilon<$ 1 in 3-methylglutaric anhydride is that the majority of crystallites have at least one spatial dimension that is very small.

The preceding discussion relates to the pure compounds. The relaxation results in the other samples of compound $\mathbf{1}$, those that have been open to the air, are more complicated. The pure sample (sample 1B1) discussed in the previous paragraph was investigated one year later (and called sample 1B2) and the temperature dependence of the various relaxation rates is shown by the eight large filled symbols in Fig. 7 where the data for the pure samples of $\mathbf{1}$ and $\mathbf{2}$ from Fig. 6 are also shown by smaller open symbols for comparison. This sample had been left open to the air (via a small hole) for one year. At temperatures above the maximum in the relaxation rates $(\omega \tau<1)$, this sample's $R_{\mathrm{S}}$ values are between the $R_{\mathrm{S}}$ values for the pure compounds. However, solid state ${ }^{1} \mathrm{H}$ spin-lattice relaxation experiments are much more sensitive to the state of the sample at temperatures below the maximum in the relaxation rate $(\omega \tau>1)$. Below approximately $170 \mathrm{~K}$, in sample $\mathbf{1 B 2}$ the relaxation is no longer exponential as found in the pure compounds. A five-parameter double exponential fits $M(t)$ very well. [See ref. 11 for a typical $M(t)$ versus $t$ plot.] The resulting value of $R_{1}$ in the double exponential fit for the three low temperature data points in Fig. 7 is indicated by downward pointing triangles. $R_{1}$ for this sample (sample 1B2) is the same as $R$ for the pure sample (sample 1B1). This suggests that this is a property of compound 1 . The other component $R_{2}$ of the double exponential that fits $M(t)$ versus $t$ (upward pointing triangles in Fig. 7) can be matched with the relaxation rates for the pure compound 2. The fractional equilibrium magnetization $\phi_{1}$ relaxing with $R_{1}$ is $40 \pm 10 \%$ and the fractional equilibrium magnetization $\phi_{2}$ relaxing with $R_{2}$ is $60 \pm 10 \%$. (See Table 1.) Presumably, in the year between using the pure sample 1B (called sample 1B1 soon after purification and sample $\mathbf{1 B 2}$ after being exposed to the air for a year) approximately half compound 1 has converted to compound 2.

To investigate this conversion phenomenon in greater detail (and to check the reproducibility of the proposed model; that the solid state ${ }^{1} \mathrm{H}$ spin-lattice relaxation experiments are observing $\mathbf{1}$ convert to $\mathbf{2}$ ) we used another sample of $\mathbf{1}$, sample $\mathbf{1 A}$. This sample was taken directly from the manufacturer's bottle without purification. We measured the temperature 
dependence of the solid state ${ }^{1} \mathrm{H}$ relaxation parameters one month after being exposed to the air (sample 1A1), one year after being exposed to the air (sample 1A2), and two years after being exposed to the air (sample 1A3). The data for these three sets of experiments is shown in Fig. 8. The relaxation rates $R_{1}$ and $R_{2}$ at low temperatures for samples $\mathbf{1 A 1}$ and $\mathbf{1 A 2}$ mirror those in sample 1B2 in Fig. 7. However, the fractional equilibrium magnetizations $\phi_{1}$ and $\phi_{2}$ are different (Table 1). In sample 1A1, the fractional equilibrium magnetizations are $\phi_{1}=50 \pm 10 \%$ corresponding to $\mathbf{1}$ and $\phi_{2}=50 \pm 10 \%$ corresponding to $\mathbf{2}$. For sample $\mathbf{1 A 2}$, however, these become $\phi_{1}=25 \pm 10 \%$ corresponding to $\mathbf{1}$ and $\phi_{2}=75 \pm 10 \%$ corresponding to 2 . In this case, more of compound $\mathbf{1}$ has converted to compound $\mathbf{2}$. The ${ }^{1} \mathrm{H}$ spin-lattice relaxation rates for sample $\mathbf{1 A 3}$ a year later (two years exposed to the air) in Fig. 8 is the same as sample $\mathbf{1 A 2}$ at higher temperatures but at lower temperatures neither a four-parameter stretched exponential or a five-parameter double exponential fit $M(t)$ versus $t$ very well. We show the $R^{*}$ (from a stretched exponential) values for sample $\mathbf{1 A 3}$ in Fig. 8 as a guide, despite the poorness of fit. Most of the sample $\mathbf{1 A 3}$ has converted to $\mathbf{2}$. The signal-to-noise in the relaxation experiments, though high, is not high enough to fit $M(t)$ versus $t$ to a double exponential if one fractional magnetization is less than approximately $10 \%$ (but well above zero). In any event, the low temperature $R^{*}$ values for sample 1A3 suggest that most of the sample has converted but enough has not that the relaxation rate data is difficult to fit. This was verified by the high resolution ${ }^{1} \mathrm{H}$ NMR spectroscopy experiment that showed that approximately $5 \%$ of this sample is $\mathbf{1}$ and approximately $95 \%$ of this sample is 2. These result are summarized in Table 1. The ${ }^{1} \mathrm{H}$ spin-lattice relaxation rate experiments in sample 1A over the two year period (three sets of fractional equilibrium magnetizations $\phi_{1}$ and $\phi_{2}$, each with a large uncertainty) provide a conversion time constant $T_{\mathrm{E}} \sim$ several months to about a year. This is consistent with the fact that this sample was open to the air via a very small hole, that the surface-to-volume ratio for the sample was very small, and that the humidity varied from $10 \%$ to $50 \%$ (i.e., much less than $87 \%$ for the experiment discussed in Section 4.1 where $T_{\mathrm{E}}$ was 24 days).

The solid state ${ }^{1} \mathrm{H}$ spin-lattice relaxation rate data in all these samples can be used to put crude limits on the manner in which compounds $\mathbf{1}$ and $\mathbf{2}$ are mixed in the samples when a fiveparameter double exponential successfully fits the time dependence of the perturbed magnetization $M(t)$ (but a four-parameter stretched exponential and a three-parameter single exponential do not). If molecules of $\mathbf{1}$ and $\mathbf{2}$ were "mixed" homogeneously on the molecular 
scale, only a single relaxation process would be found. That is, even though there are two different 'species' of methyl groups (those on molecules of $\mathbf{1}$ and those on molecules of $\mathbf{2}$ ) with two different correlation times $\tau_{1}$ and $\tau_{2},{ }^{1} \mathrm{H}-{ }^{1} \mathrm{H}$ spin diffusion would result in a single average spin-lattice relaxation process. The spin diffusion process (with a little help from lattice vibrations) involves energy conserving mutual spin flips and can redistribute energy rapidly over at least several molecules. This process occurs on a time scale that is characterized by the inverse of the solid state ${ }^{1} \mathrm{H}$ NMR line width which, for these samples is in the range of spin-spin relaxation times $T_{2}=8-15 \mu \mathrm{s}$ (depending slightly on temperature). This is to be compared with the spin-lattice relaxation times $T_{1}=R^{-1}$ which (Fig. 6) are $20 \mathrm{~ms}-4 \mathrm{~s}$, many orders of magnitude slower. So, as the $\mathrm{CH}_{3}{ }^{1} \mathrm{H}$ spins relax in the spin-lattice relaxation process, they rapidly perform energy conserving spin flips with nearby non $\mathrm{CH}_{3}{ }^{1} \mathrm{H}$ spins via the spin-spin relaxation process and thus maintain a constant spin temperature. (This is the origin of the factor $n / N$ in $R=(n / N)(1$ $+\mathrm{z}) A_{\text {intraCH3 } 3}[J(\omega, \tau)+4 J(2 \omega, \tau)]$. $)$ But this is a through-space process and the presence of a double exponential clearly indicates that the two parts of the sample (molecules of $\mathbf{1}$ and $\mathbf{2}$ ) are not "communicating" with each other via this mechanism. This would happen if one literally took two pure macroscopic samples and physically mixed the two solids. This is what the solid state ${ }^{1} \mathrm{H}$ spin-lattice relaxation experiments are mimicking in cases where the double exponential fits are successful. In the present case the anhydride (1) is absorbing water and turning into the acid (2). And this is not occurring in a spatially homogeneous manner. It is occurring in volumes of the sample that involve at least several molecules and probably entire very small crystallites.

Finally, we show the temperature dependence of $\beta$ in the stretched exponential fits of $M(t)$ versus $t$ for all eight distinct NMR samples for both compounds (Table 1) in Fig. 9. The parameter $\beta$ is plotted versus $T^{1}-T^{1}{ }_{\text {max }}$ where $T_{\max }$ is the temperature of the relaxation rate maximum which, as indicated in Fig 6, is $160 \mathrm{~K}$ for $\mathbf{1}$ and $195 \mathrm{~K}$ for $\mathbf{2}$. We plot $\beta$ versus $T^{1}-T$ ${ }^{1}{ }_{\max }$ (rather than just $T^{1}$ ) because the degree of nonexponential relaxation (characterized by $\beta$ ) correlates with the maximum in the relaxation rate (as discussed in section 3). This graph can be compared with Fig. 1 in ref. 10 which shows a similar plot for six very different organic solids with methyl groups. Even though $\beta$ versus $T^{1}-T^{1}{ }_{\text {max }}$ is not amenable to interpretation in terms of a fundamental closed-form model, it is, nevertheless, a signature of $\mathrm{CH}_{3}$ rotation, at least in a large class of organic van der Waals solids [10]. Note that $\beta$ versus $T^{-1}-T^{1}{ }_{\max }$ for the two pure 
samples of $\mathbf{1}$ and $\mathbf{2}$ covers the entire temperature range (for each compound) whereas for all the other samples of $\mathbf{1}$ (with significant amounts of $\mathbf{2}$ in the sample), $\beta$ is only plotted above 160-200 $\mathrm{K}$ (depending on sample) because the low temperature $M(t)$ versus $t$ for these samples cannot be fitted by a stretched exponential. A double exponential was required.

\section{Discussion}

The NMR activation energy for methyl group rotation is $12 \pm 1 \mathrm{~kJ} \mathrm{~mole}^{-1}$ in pure solid 3methylglutaric anhydride (1) and $16 \pm 2 \mathrm{~kJ} \mathrm{~mole}^{-1}$ in pure solid 3-methylglutaric acid (2). The calculated values for the methyl group rotational barrier for a molecule in the center of a cluster built from the X-ray structures are $16 \mathrm{~kJ} \mathrm{~mole}^{-1}$ in $\mathbf{1}$ and $20 \mathrm{~kJ} \mathrm{~mole}^{-1}$ in $\mathbf{2}$. Both the measured NMR activation energies in the solid samples and the calculated barriers in the clusters built from the X-ray structure automatically include contributions from both intramolecular and intermolecular interactions. With the understanding that the NMR activation energy and the computed barrier, though closely related, are not exactly the same [50,51], these values are in reasonable, but not excellent, agreement. One does, however, expect the NMR activation energy to be somewhat smaller than the computed barrier [11 50-51].

Insofar as the van der Waals intermolecular interactions will change the structure of the covalently bonded molecules very slightly, separating the total contributions to the methyl group rotational barrier into intramolecular and intermolecular components is not well-defined. However, as a rough guide, we can consider the electronic structure barrier calculations by themselves, independently of any experiment. These barriers are obtained by subtracting two very large single-molecule or entire-cluster energies; one energy with the methyl group in the ground state and another energy with the methyl group in a transition state. These calculations for single small molecules are now routine and very accurate. The computed values of the methyl group barriers in the isolated molecules are $13.1 \mathrm{~kJ} \mathrm{~mole}^{-1}$ in $\mathbf{1}$ and $13.8 \mathrm{~kJ} \mathrm{~mol}^{-1}$ in $\mathbf{2}$. It is reasonable to attach an uncertainty of only $\pm 0.5 \mathrm{~kJ} \mathrm{~mol}^{-1}$ to these calculated barriers. The computed barriers in the clusters are $16 \mathrm{~kJ} \mathrm{~mol}^{-1}$ in $\mathbf{1}$ and $20 \mathrm{~kJ} \mathrm{~mol}^{-1}$ in $\mathbf{2}$. These calculations are challenging and still a very active area of research. It is reasonable to attach an uncertainty of \pm 1 $\mathrm{kJ} \mathrm{mol}^{-1}$ to these barriers. We can conclude that the computed contribution (to the methyl group barrier) of the intermolecular potentials are approximately $(16 \pm 1)-(13.1 \pm 0.5)=3 \pm 2 \mathrm{~kJ} \mathrm{~mol}^{-1}$ 
in $\mathbf{1}$ and $(20 \pm 1)-(13.8 \pm 0.5)=6 \pm 2 \mathrm{~kJ} \mathrm{~mol}^{-1}$ in $\mathbf{2}$. These values can be compared with similar values in related compounds [11].

A useful parameter that characterizes a plot of the logarithm of the solid state ${ }^{1} \mathrm{H}$ spinlattice relaxation rate as a function of the inverse temperature is the ratio of the magnitude of the slope at low temperature to the magnitude of the slope at high temperature. A value of unity for this parameter in pure 3-methylglutaric acid suggests a unique value for the methyl group rotational NMR activation energy which, in turn, suggests that a vanishingly small fraction of molecules reside on crystallite surfaces or near other crystal imperfections. This is consistent with the large crystallites observed in the field emission scanning electron microscopy images. For pure 3-methylglutaric anhydride, however, this ratio of slopes is 0.8 which implies an observable distribution of methyl group rotational NMR activation energies resulting from the fact that methyl groups in molecules on crystallite surfaces or near other crystal imperfections will have a different barrier from methyl groups in the bulk crystal environment. This is consistent with the field emission scanning electron microscopy images that suggest there is a large variation in sample morphology and that there are many very small particles which may or may not be single crystallites, even at the hundreds of nanometers scale.

The preceding comments refer to the pure compounds. We have also observed ${ }^{1} \mathrm{H}$ spinlattice relaxation rates in samples of 3-methylglutaric anhydride that are absorbing water and converting to 3-methylglutaric acid $\left[\mathrm{C}_{6} \mathrm{H}_{8} \mathrm{O}_{3}(\mathbf{1})+\mathrm{H}_{2} \mathrm{O} \rightarrow \mathrm{C}_{6} \mathrm{H}_{10} \mathrm{O}_{4}(\mathbf{2})\right]$. ${ }^{1} \mathrm{H}-{ }^{1} \mathrm{H}$ spin diffusion between the ${ }^{1} \mathrm{H}$ spins in methyl groups and nearby ${ }^{1} \mathrm{H}$ spins is fast enough to ensure a common spin temperature among spins in molecules that are nearby. But the observation that a double exponential is the simplest model that fits a relaxing ${ }^{1} \mathrm{H}$ magnetization following a perturbation, with the two relaxation rates characteristic of each compound, shows unequivocally that ${ }^{1} \mathrm{H}$ spin diffusion between the two compounds in the sample is not occurring. This implies that there are clusters of molecules of each compound. One suspects that whole very small particles of 3methylglutaric anhydride are absorbing water and turning into 3-methylglutaric acid. If this hydrolysis process were occurring in a spatially homogenous manner, spin diffusion would not result in a perturbed ${ }^{1} \mathrm{H}$ nuclear magnetization relaxing via a double exponential. 
[1] R. Kimmich, NMR Tomography, Diffusometry, Relaxometry, Springer-Verlag, Berlin, 1997.

[2] P. A. Beckmann, C. W. Mallory, F. B. Mallory, A. L. Rheingold, and X. Wang, ChemPhysChem 16 (2015) 1509-1519.

[3] J. Goldstein, D. E. Newbury, D. C. Joy, C. E. Lyman, P. Echlin, E. Lifshin, L. Sawyer, and J. R. Michael, Scanning Electron Microscopy and X-ray Microanalysis, 3rd ed., Springer Science + Business Media, New York, 2003.

[4] G. W. H. Höhne, W. F. Hemmingger, H. -J. Flammersheim, Differential Scanning Calorimetry, 2nd ed., Springer-Verlag, Berlin, 2003.

[5] J. Kohanoff, Electronic Structure Calculations for Solids and Molecules: Theory and Computational Methods, Cambridge Univ. Press, Cambridge, UK, 2006.

[6] R. Tilley, Crystals and Crystal Structures, Wiley, Chichester, UK, 2006.

[7] P. A. Beckmann, K. G. Conn, C. W. Mallory, F. B. Mallory, A. L. Rheingold, L. Rotkina, and X. Wang, J. Chem. Phys. 139 (2013) 204501, 1-12.

[8] L. K. Runnells, Phys. Rev. 134 (1964) A28-A36.

[9] R. L. Hilt and P. S. Hubbard, Phys. Rev. 134 (1964) A392-A398.

[10] P. A. Beckmann, Solid State Nuc. Mag. Resonan. 71 (2015) 91-95.

[11] P. A. Beckmann and A. L. Rheingold, J. Chem. Phys. 144 (2016) 154308, 1-12.

[12] S. R. Chaudhari, Chem. Phys. Lett. 634 (2015) 95-97.

[13] T. M. B. Islam, K. Yoshino, H. Nomura, T. Mizuno, and A. Sasane, Anal. Sci. 18 (2002) 363-366.

[14] C. H. L. Goodman, Crystal Growth Theory and Techniques Volume 1, Plenum, London, 1974.

[15] Gaussian 09, Revision D.01, M. J. Frisch, G. W. Trucks, H. B. Schlegel, G. E. Scuseria, M. A. Robb, J. R. Cheeseman, G. Scalmani, V. Barone, B. Mennucci, G. A. Petersson, H. Nakatsuji, M. Caricato, X. Li, H. P. Hratchian, A. F. Izmaylov, J. Bloino, G. Zheng, J. L. Sonnenberg, M. Hada, M. Ehara, K. Toyota, R. Fukuda, J. Hasegawa, M. Ishida, T. Nakajima, Y. Honda, O. Kitao, H. Nakai, T. Vreven, J. A. Montgomery, Jr., J. E. Peralta, F. Ogliaro, M. Bearpark, J. J. Heyd, E. Brothers, K. N. Kudin, V. N. Staroverov, R. Kobayashi, J. Normand, K. Raghavachari, A. Rendell, J. C. Burant, S. S. Iyengar, J. Tomasi, M. Cossi, N. Rega, J. M. Millam, M. Klene, J. E. Knox, J. B. Cross, V. Bakken, C. Adamo, J. Jaramillo, R. Gomperts, R. E. Stratmann, O. Yazyev, A. J. Austin, R. Cammi, C. Pomelli, J. W. Ochterski, R. L. Martin, K. Morokuma, V. G. Zakrzewski, G. A. Voth, P. Salvador, J. J. Dannenberg, S. Dapprich, A. D. Daniels, Ö. Farkas, J. B. Foresman, J. V. Ortiz, J. Cioslowski, D. J. Fox, Gaussian, Inc., Wallingford CT, 2009.

[16] X. Wang, F. B. Mallory, C. W. Mallory, H. R. Odhner, and P. A. Beckmann, J. Chem. Phys. 140 (2014) 194304, 1-15.

[17] V. L. Deringer, V. Hoepfner, and R. Dronskowski, Cryst. Growth Des. 12 (2012) 10141021. 
[18] T. Steiner and W. Saenger, Acta Cryst. A49 (1993) 379-384.

[19] H. Kruse and S. Grimme, J. Chem. Phys. 136 (2012) 1-16.

[20] S. Grimme, WIREs Comp. Mol. Sci. 1 (2011) 211-228.

[21] E. O. Stejskal and H. S. Gutowsky, J. Chem. Phys. 28 (1958) 388-396. The factor 3/40 in equation 21 should be $3 / 20$.

[22] P. A. Beckmann and E. Schneider, J. Chem. Phys. 136 (2012) 054508, 1-9.

[23] E. R. Andrew, R. Gaspar Jr., and W. Vennart, Chem. Phys. Lett. 38 (1976) 41-43.

[24] K. van Putte, J. Mag. Resonan. 5 (1975) 367-375.

[25] J. D. Cutnell and L. Verduin, J. Chem. Phys. 59 (1973) 258-262.

[26] J. D. Cutnell and W. Venable, J. Chem. Phys. 60 (1974) 3795-3801.

[27] M. Mehring and H. Raber, J. Chem. Phys. 59 (1973) 1116-1120.

[28] M. F. Baud and P. S. Hubbard, Phys. Rev. 170 (1968) 384-390.

[29] R. Kohlrausch, Ann. Phys. Chem. (Poggendorff) 91 (1854) 179-214.

[30] M. Berberan-Santos, E. N. Bodunov, and B. Valeur, Ann. Phys. (Berlin) 17 (2008) 460-461.

[31] R. M. Pickup, R. Cywinski, C. Pappas, B. Farago, and P. Fouquet, Phys. Rev. Lett. 102 (2009) 097202, 1-4 . Reference 1 in this paper is incorrect. It should be reference 29 in this work.

[32] C. M. Roland and K. L. Ngai, J. Chem. Phys. 103 (1995) 1152-1159.

[33] J. G. Powles, D. M. Heyes, G. Rickayzen, and W. A. B. Evans, J. Chem. Phys. 131 (2009) 214509, 1-8.

[34] M. N. Berberan-Santos, Chem. Phys. Lett. 460 (2008) 146-150.

[35] M. N. Berberan-Santos, E. N. Bodunov, and B. Valeur, Chem. Phys. 315 (2005) 171-182.

[36] D. W. Davidson and R. H. Cole, J. Chem. Phys. 19 (1951) 1484-1490.

[37] P. A. Beckmann, L. Happersett, A. V. Herzog, and W. M. Tong, J. Chem. Phys. 95 (1991) 828-835.

[38] S. Clough and A. Heidemann, J. Phys. C: Solid State Phys. 13 (1980) 3585-3589.

[39] S. Clough, A. Heidemann, A. J. Horsewill, J. D. Lewis, and M. N. J. Paley, J. Phys. C: Solid State Phys. 14 (1981) L525-L529.

[40] S. Clough and P. J. McDonald, J. Phys. C: Solid State Phys. 15 (1982) L1039-L1042.

[41] S. Clough, P. J. McDonald, and F. O. Zelaya, J. Phys. C: Solid State Phys. 17 (1984) 44134420 .

[42] D. Cavagnat, S. Clough, F. O. Zelaya, J. Phys. C: Solid State Phys. 18 (1985) 6457-6462.

[43] S. Clough, Physica 136B (1986) 145-149.

[44] M. J. Barlow, S. Clough, A. J. Horsewill, and M. A. Mohammed, Solid State Nuc. Mag. Resonan. 1 (1992) 197-204. 
[45] S. Clough, Solid State Nuc. Mag. Resonan. 9 (1997) 49-53.

[46] J. S. Waugh and É. I. Fedin, Soviet Physics - Solid State 4 (1963) 1633-1636.

[47] R. Ferrando, R. Spadacini, G. E. Tommei, and V. I. Mel'nikov, Phys. Rev. E 51 (1995) R1645-R1648.

[48] N. L. Owen, in Internal Rotation in Molecules, edited by W. J. Orville-Thomas, Wiley, New York, 1974.

[49] E. K. van Putte, J. Mag. Resonan. 2 (1970) 216-225.

[50] J. Kowaleski and T. Liljefors, Chem. Phys. Lett. 64 (1979) 170-174.

[51] O. Edholm and C. Blomberg, Chem. Phys. 56 (1981) 9-14.

[52] D. Michel and V. Rossinger, J. Mag. Resonan. 28 (1977) 235-242.

[53] C. Palmer, A. M. Albano, and P. A. Beckmann, Physica B 190 (1993) 267-284.

[54] P. A. Beckmann, J. Rosenberg, K. Nordstrom, C. W. Mallory, and F. B. Mallory, J. Phys. Chem. A 110 (2006) 3947-3953.

[55] C. Kittel, Introduction to Solid State Physics, 8th ed., Wiley, USA, 2005. 
Table 1. The various samples used in the solid state ${ }^{1} \mathrm{H}$ NMR relaxation experiments.

\begin{tabular}{|c|c|c|c|}
\hline Sample & $\begin{array}{l}\text { Time } \\
\text { label }\end{array}$ & Information & $\begin{array}{l}\text { Sample composition } \\
\text { based on }{ }^{1} \mathrm{H} \\
\text { relaxation } \mathrm{NMR}\end{array}$ \\
\hline \multirow[t]{3}{*}{$1 \mathrm{~A}$} & $1 \mathrm{~A} 1$ & $\begin{array}{l}1 \text { from supplier } \\
\text { open to air }{ }^{\mathrm{a}} \text { for one month } \\
\text { experiments performed over two months }\end{array}$ & $\begin{array}{l}50 \pm 10 \% \mathbf{1} \\
50 \pm 10 \% \mathbf{2}\end{array}$ \\
\hline & $1 \mathrm{~A} 2$ & $\begin{array}{l}\text { open to air }{ }^{\mathrm{a}} \text { for } 1 \text { year } \\
\text { experiments performed over three months }\end{array}$ & $\begin{array}{l}25 \pm 10 \% \mathbf{1} \\
75 \pm 10 \% \mathbf{2}\end{array}$ \\
\hline & $1 \mathrm{~A} 3$ & $\begin{array}{l}\text { open to air }{ }^{\mathrm{a}} \text { for } 2 \text { years } \\
\text { experiments performed over three months }\end{array}$ & $\begin{array}{l}<10 \% \mathbf{1}^{\mathrm{b}} \\
>90 \% \mathbf{2}^{\mathrm{b}}\end{array}$ \\
\hline \multirow[t]{2}{*}{$1 \mathrm{~B}$} & 1B1 & $\begin{array}{l}1 \text { purified by zone refinement } \\
\text { open to air }{ }^{\mathrm{a}} \text { for one hour } \\
\text { experiments performed over one month }\end{array}$ & $100 \% 1$ \\
\hline & 1B2 & $\begin{array}{l}\text { open to air }{ }^{\mathrm{a}} \text { for one year } \\
\text { experiments performed over three months }\end{array}$ & $\begin{array}{l}40 \pm 10 \% \mathbf{1} \\
60 \pm 10 \% \mathbf{2}\end{array}$ \\
\hline \multirow[t]{2}{*}{$2 \mathrm{~A}$} & $2 \mathrm{~A} 1$ & $\begin{array}{l}2 \text { from supplier } \\
\text { open to air }{ }^{\mathrm{a}} \text { for one hour } \\
\text { experiments performed over three months }\end{array}$ & $100 \% 2$ \\
\hline & $2 \mathrm{~A} 2$ & $\begin{array}{l}\text { open to air }{ }^{\mathrm{a}} \text { for one year } \\
\text { experiments performed over one month }\end{array}$ & $\begin{array}{l}100 \% 2 \\
\text { no change from } 2 \mathrm{~A} 1\end{array}$ \\
\hline $2 \mathrm{~B}$ & $2 \mathrm{~B} 1$ & $\begin{array}{l}2 \text { purified by recrystallization } \\
\text { open to air }{ }^{\mathrm{a}} \text { for one hour } \\
\text { experiments performed over one month }\end{array}$ & $\begin{array}{l}100 \% \mathbf{2} \\
\text { no different than } 2 \mathrm{~A} 1\end{array}$ \\
\hline
\end{tabular}

${ }^{\text {a }}$ There was a small hole (for the thermocouple) through a Teflon tape "seal" at the end of the NMR sample tube.

${ }^{\mathrm{b}}$ Confirmed by high-resolution ${ }^{1} \mathrm{H}$ NMR spectroscopy. 
Table 2. X-ray diffraction data for pure samples of $\mathbf{1}$ and $\mathbf{2}$

\begin{tabular}{|c|c|c|}
\hline & 3-methylglutaric anhydride (1) & 3-methylglutaric acid (2) \\
\hline CSD deposit number & 1477660 & 1477661 \\
\hline Empirical formula & $\mathrm{C}_{6} \mathrm{H}_{8} \mathrm{O}_{3}$ & $\mathrm{C}_{6} \mathrm{H}_{10} \mathrm{O}_{4}$ \\
\hline Formula weight & 128.12 & 146.14 \\
\hline Temperature & $100(2) \mathrm{K}$ & $100(2) \mathrm{K}$ \\
\hline Wavelength & $0.71073 \AA$ & $0.71073 \AA$ \\
\hline Crystal system & Monoclinic & Monoclinic \\
\hline Space group & $C 2 / c$ & $P 2{ }_{1} / c$ \\
\hline \multirow[t]{6}{*}{ Unit cell dimensions } & $a=20.878(5) \AA$ & $a=13.795(6) \AA$ \\
\hline & $b=5.8522(12) \AA$ & $b=5.313(3) \AA$ \\
\hline & $c=12.293(3) \AA$ & $c=10.073(4) \AA$ \\
\hline & $\left\langle=90^{\circ}\right.$ & $\alpha=90^{\circ}$ \\
\hline & $(B)=122.756(12)^{\circ}$ & $\beta=109.745(12)^{\circ}$ \\
\hline & $(C)=90^{\circ}$ & $\gamma=90^{\circ}$ \\
\hline Volume & $1263.2(5) \AA^{3}$ & $694.9(6) \AA^{3}$ \\
\hline$Z$ & 8 & 4 \\
\hline$Z$ & 1 & 1 \\
\hline Density (calculated) & $1.347 \mathrm{Mg} / \mathrm{m}^{3}$ & $1.397 \mathrm{Mg} / \mathrm{m}^{3}$ \\
\hline Absorption coefficient & $0.109 \mathrm{~mm}^{-1}$ & $0.118 \mathrm{~mm}^{-1}$ \\
\hline$F(000)$ & 544 & 312 \\
\hline Crystal size & $0.38 \times 0.33 \times 0.30 \mathrm{~mm}^{3}$ & $0.40 \times 0.38 \times 0.090 \mathrm{~mm}^{3}$ \\
\hline Theta range for data collection & 3.320 to $25.348^{\circ}$ & 3.138 to $28.273^{\circ}$ \\
\hline Index ranges & $\begin{array}{l}-24 \leq h \leq 24,-7 \leq k \leq 7 \\
-14 \leq l \leq 14\end{array}$ & $\begin{array}{l}-18 \leq h \leq 17,0 \leq k \leq 7 \\
0 \leq l \leq 13\end{array}$ \\
\hline Reflections collected & 5815 & 10576 \\
\hline Independent reflections & $1153[R($ int $)=0.0261]$ & $1717[R($ int $)=0.0385]$ \\
\hline Completeness to theta $=25.242^{\circ}$ & $99.8 \%$ & $99.9 \%$ \\
\hline Absorption correction & Multi-scan & Semi-empirical from equivalents \\
\hline Refinement method & Full-matrix least-squares on $F^{2}$ & Full-matrix least-squares on $F^{2}$ \\
\hline Data / restraints / parameters & 1153 / 0 / 91 & $1717 / 0 / 97$ \\
\hline Goodness-of-fit on $F^{2}$ & 1.096 & 1.041 \\
\hline Final $R$ indices $[I>2 \operatorname{sigma}(I)]$ & $R 1=0.0503, w R 2=0.1338$ & $R 1=0.0542, w R 2=0.1499$ \\
\hline$R$ indices (all data) & $R 1=0.0621, w R 2=0.1444$ & $R 1=0.0580, w R 2=0.1560$ \\
\hline Extinction coefficient & $\mathrm{n} / \mathrm{a}$ & $\mathrm{n} / \mathrm{a}$ \\
\hline Largest diff. peak and hole & 0.473 and $-0.199 e \AA^{-3}$ & 0.430 and $-0.278 e \AA^{-3}$ \\
\hline Twinning & $\mathrm{n} / \mathrm{a}$ & $15 \% 180^{\circ}$ rot twin \\
\hline
\end{tabular}



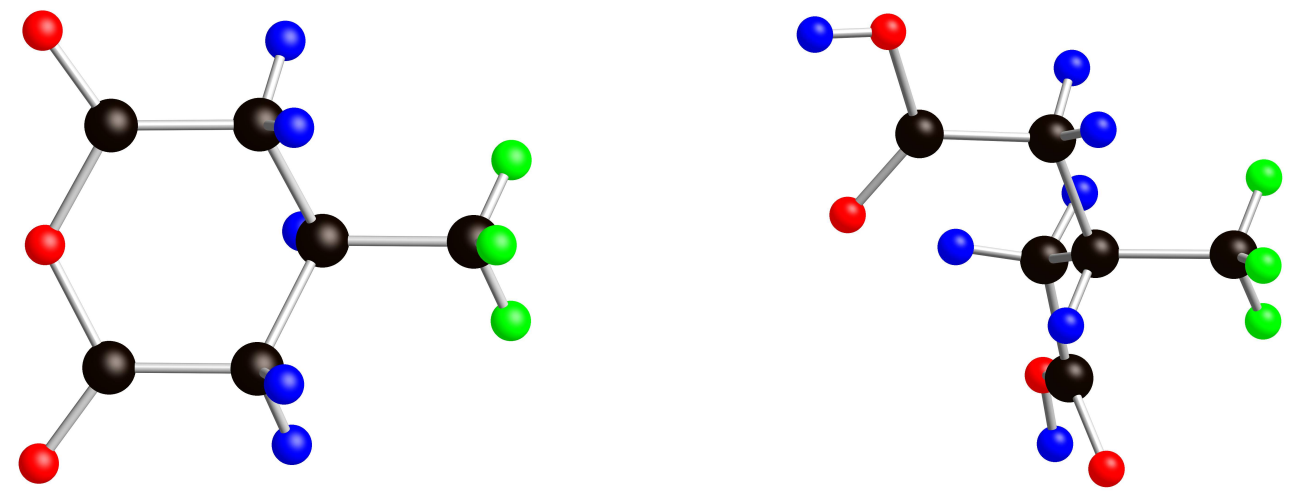
(a)

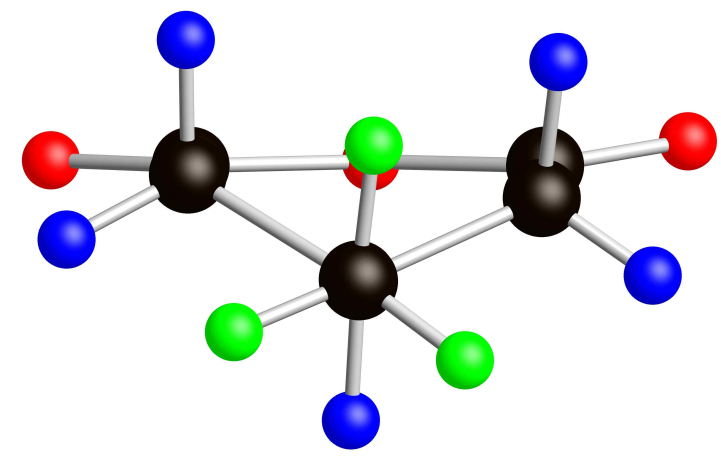

(c) (b)

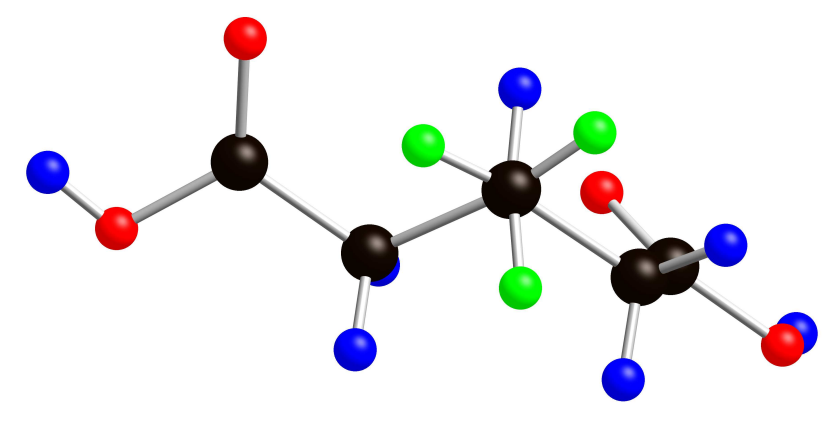

(d)

Fig. 1. (a) and (c); Two views of a molecule of 3-methylglutaric anhydride (1). (b) and (d); Two views of a molecule of 3-methylglutaric acid (2). These are the structures of the molecules in the pure crystals. At the resolution shown, the structures of the isolated molecules are the same. The asymmetric unit for both $\mathbf{1}$ and $\mathbf{2}$ is a single molecule $(Z=1)$. $\mathrm{O}$ atoms are red, $\mathrm{C}$ atoms are black, $\mathrm{CH}_{3} \mathrm{H}$ atoms are green, and all other $\mathrm{H}$ atoms are blue. (a) and (b) show a view with the $\mathrm{CH}_{3}$ rotation axis in the plane of the page (horizontally) and the three $\mathrm{CH}_{3} \mathrm{H}$ atoms in a vertical plane perpendicular to the page. (c) and (d) show a view with the $\mathrm{CH}_{3}$ rotation axes perpendicular to the plane of the page and the three $\mathrm{CH}_{3} \mathrm{H}$ atoms in the plane of the page. 


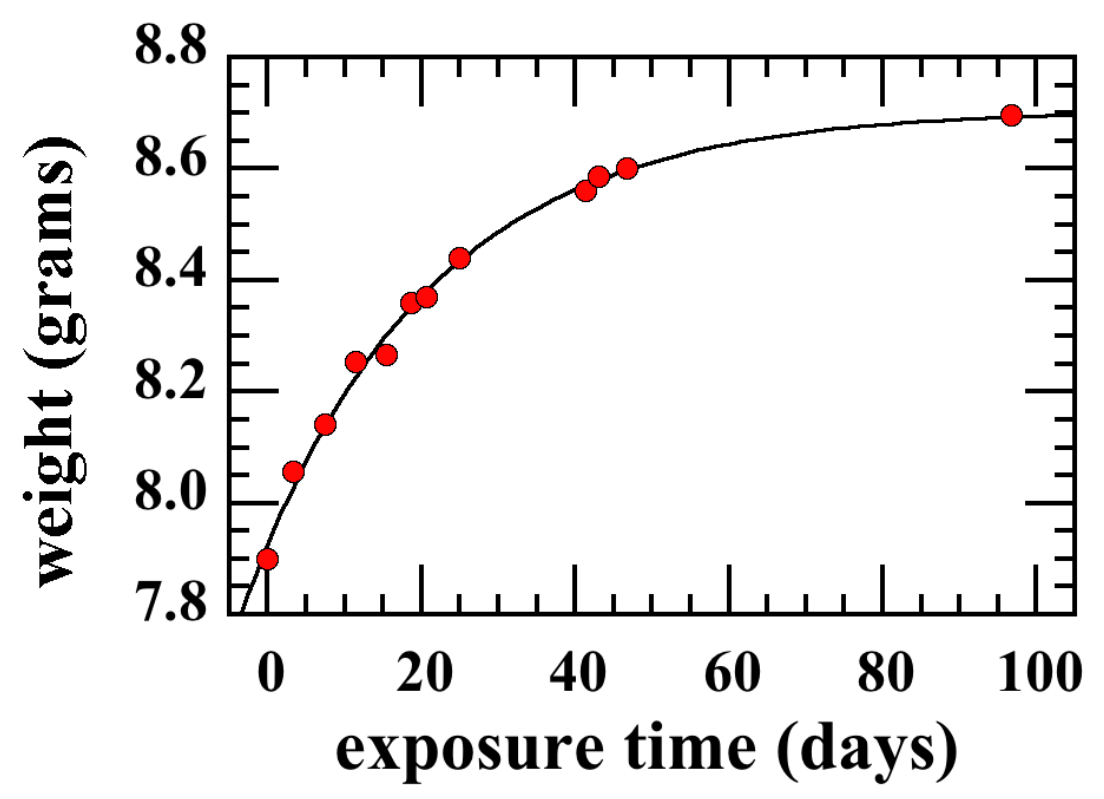

Fig. 2. The weight of a sample of 3-methylglutaric anhydride (1) as a function of time as it absorbs water and turns into 3-methylglutaric acid (2) in a constant $87 \%$ relative humidity environment. The fitted exponential is discussed in the text. 


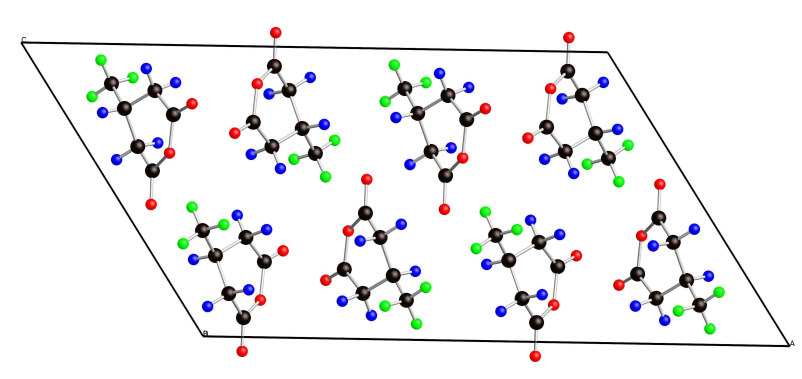

(a)

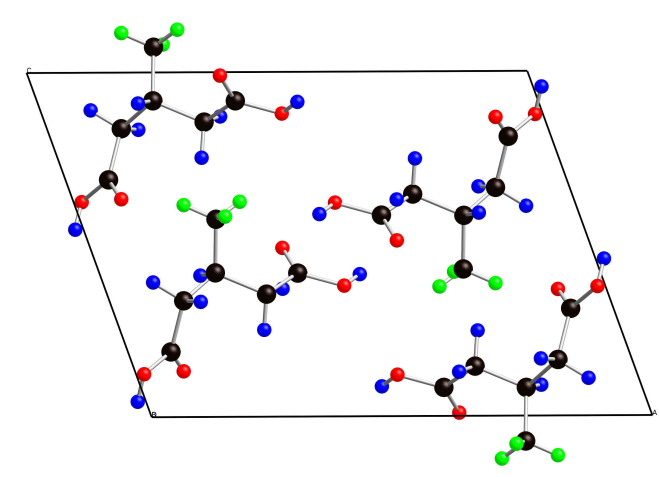

(b)

Fig. 3. The 010 plane of the crystal structure of (a) 3-methylglutaric anhydride (1) and (b) 3methylglutaric acid (2). $\mathrm{O}$ atoms are red, $\mathrm{C}$ atoms are black, $\mathrm{CH}_{3} \mathrm{H}$ atoms are green, and all other $\mathrm{H}$ atoms are blue. Crystal data and structure refinements are provided in Table 2. The lines indicate the unit cell. The two parts are not to the same scale. For (a), the unit cell dimensions shown are 2.1 (horizontal) by $1.2 \mathrm{~nm}$ and for (b) they are 1.4 (horizontal) by $1.0 \mathrm{~nm}$. Both crystal systems are monoclinic and the third dimension is $0.58 \mathrm{~nm}$ for (a) and $0.53 \mathrm{~nm}$ for (b), both perpendicular to the page. There are eight molecules per unit cell for $(\mathbf{a})(Z=8)$ and four $(Z=4)$ for (b). Only complete molecules are shown. Where atoms extend outside the unit cell, equivalent atoms (not shown) enter at the opposite face. Both structures have a single molecule as the asymmetric unit $\left(Z^{\prime}=1\right)$. 


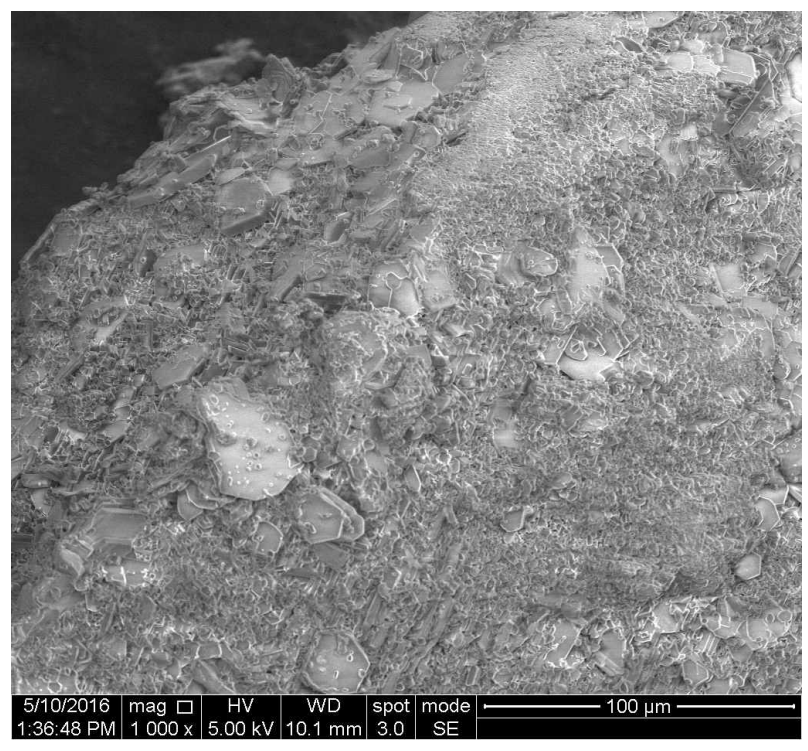

(a)

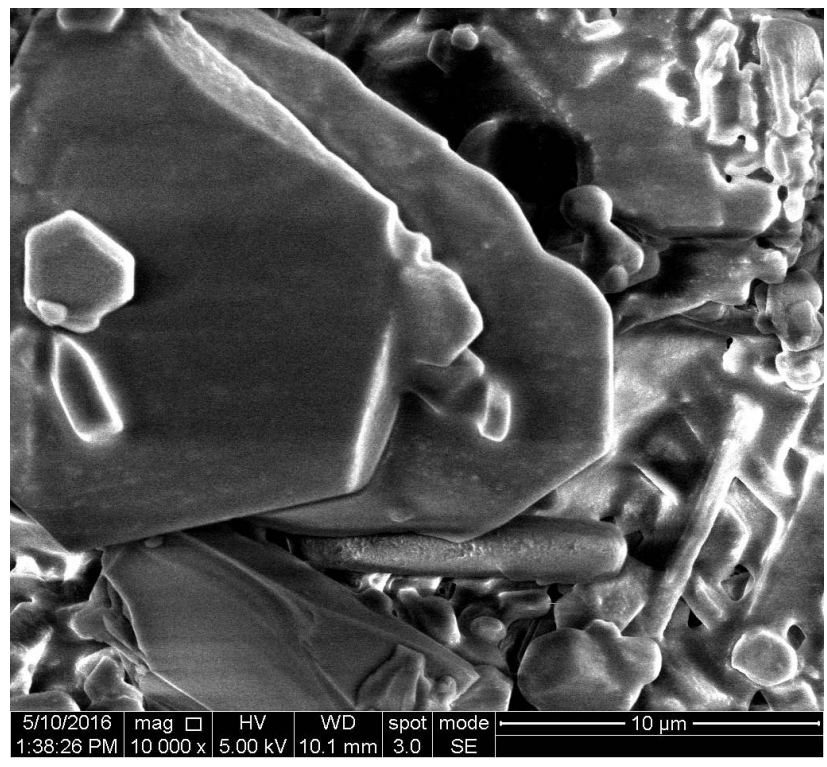

(c)

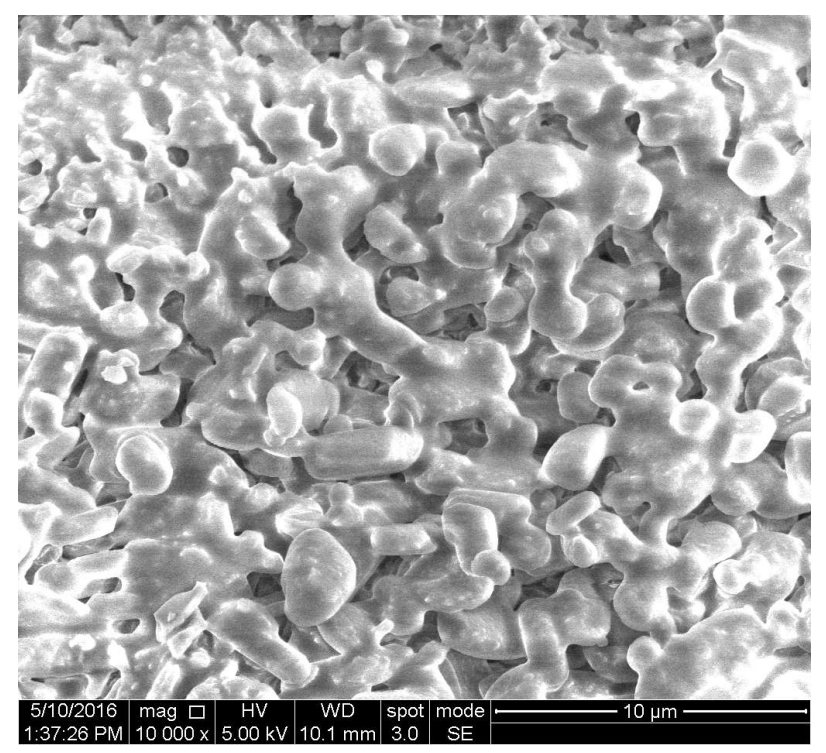

(b)

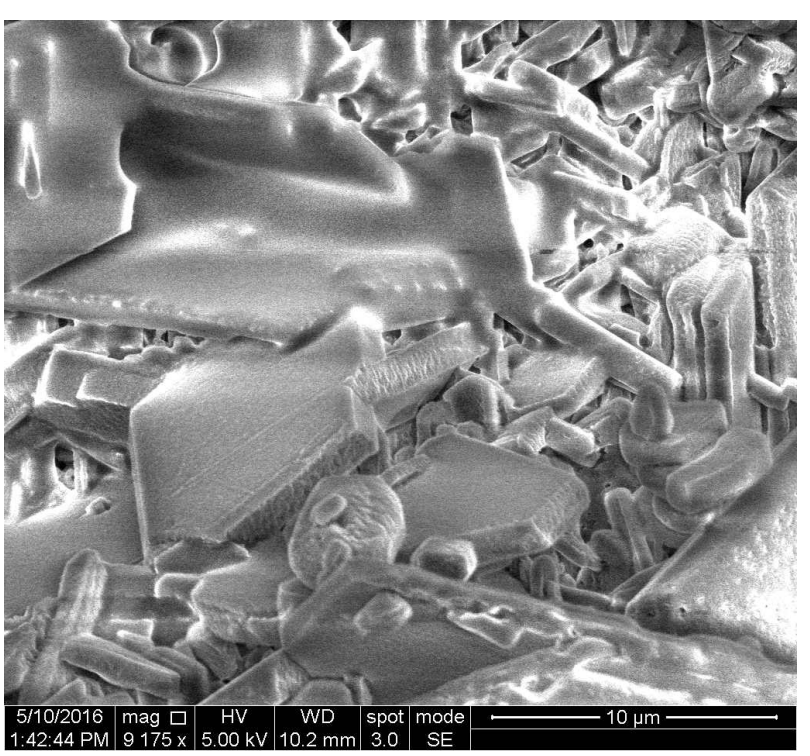

(d) 
Fig. 4. (a) A 253 X $218 \mu \mathrm{m}$ field emission scanning electron microscopy image of sample 1B1, a purified sample of 3-methylglutaric anhydride (1) open to the air for only minutes before the image was taken. Virtually no crystalline structure is discernible. (b), (c), and (d), three 25 X 22 $\mu \mathrm{m}$ images of different parts of (a) showing the large variability of morphologies and particle sizes. Of note is the preponderance of very small particles, which may or may not be single crystallites. There was some melting caused by the electron beam at the scales of (b), (c), and (d). Note the similarity of the larger crystallites in (c) to that for 3-methylglutaric acid (2) in Fig. 5 (b). The horizontal striations in (c) and (d) are an imaging artifact. 


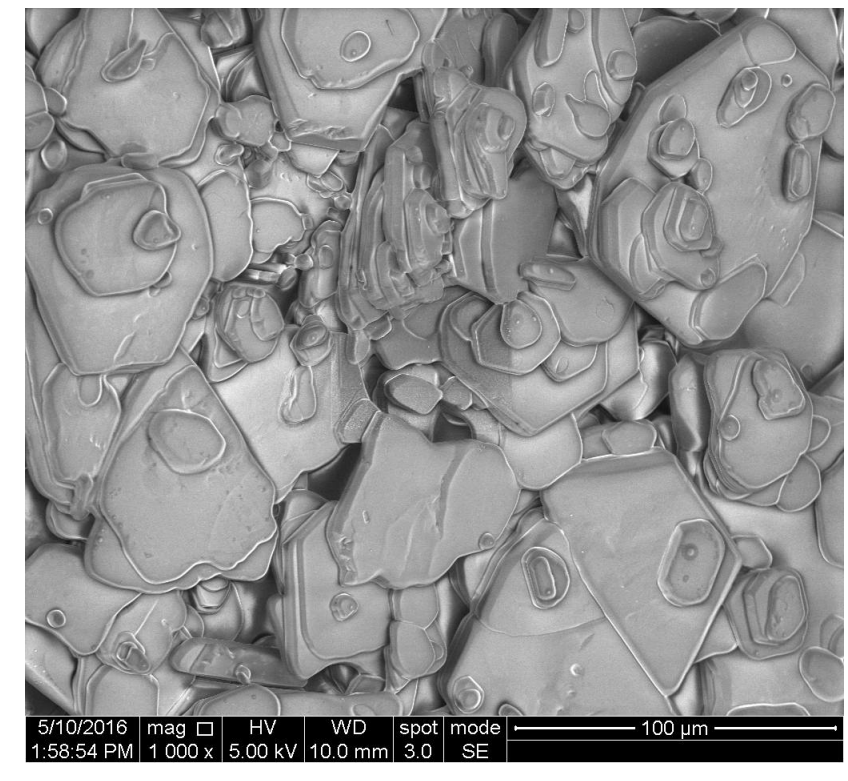

(a)

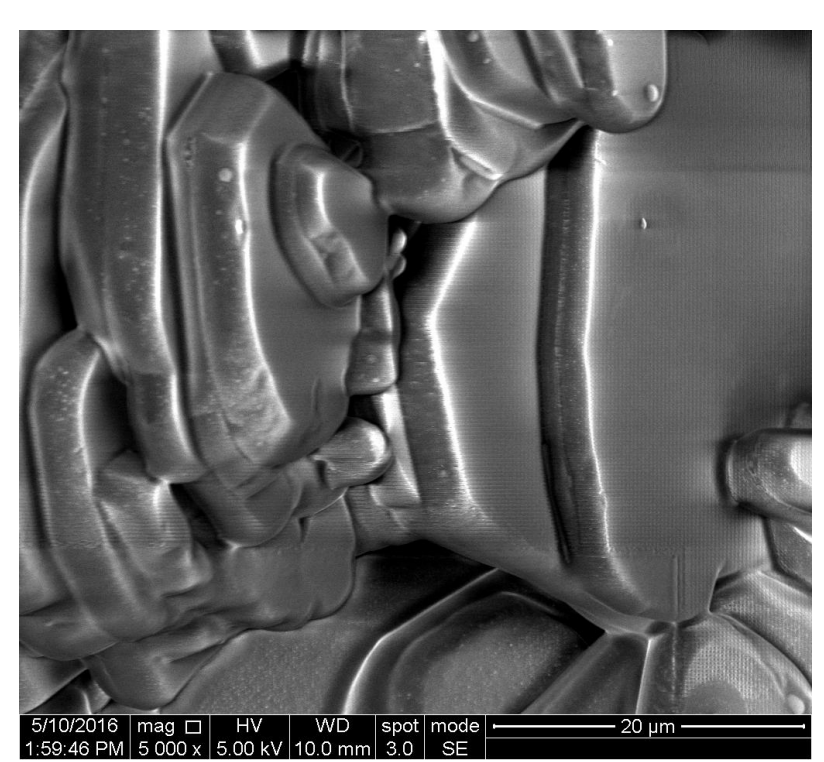

(b)

Fig. 5. (a) A 253 X $218 \mu \mathrm{m}$ field emission scanning electron microscopy image of sample 2B1, a purified sample of 3-methylglutaric acid (2). (b) a 25 X $22 \mu \mathrm{m}$ image of a part of the sample that captures some crystals on end, thus displaying the smallest dimension of the crystals. Other images with the same resolution in other parts of the sample are very similar to the images in (a) and (b). The smallest dimension of the smooth crystallites is greater than approximately $1 \mu \mathrm{m}$. No melting was caused by the electron beam. The horizontal striations in (b) are an imaging artifact. 


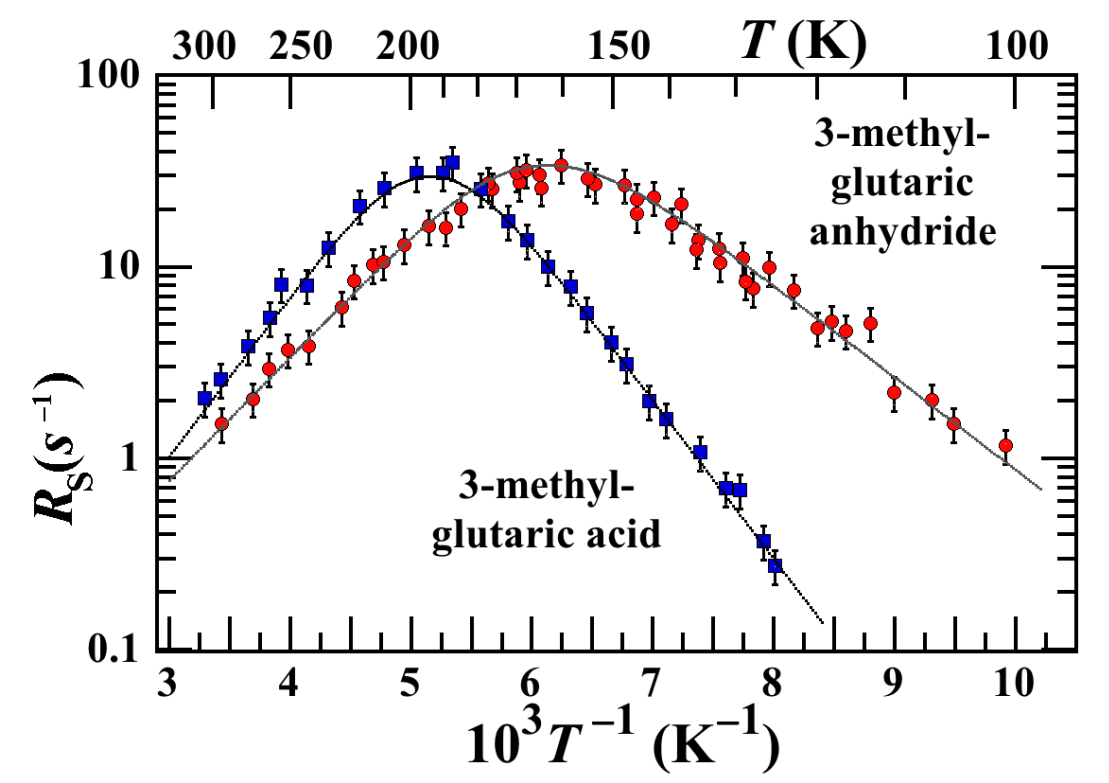

Fig. 6. The temperature $T$ dependence of the solid state ${ }^{1} \mathrm{H}$ spin-lattice initial relaxation rate $R_{\mathrm{S}}$ at 22.5 MHz in sample 1B1 of 3-methylglutaric anhydride (1) (O) and sample 2B1 of 3-

methylglutaric acid (2) (ם). These are the pure samples. At lower temperatures (below $140 \mathrm{~K}$ in 1 and below $170 \mathrm{~K}$ in 2 ) the relaxation is exponential to within experimental uncertainty and $R_{\mathrm{S}}=$ $R$, a single relaxation rate. The fits are discussed in the text. 


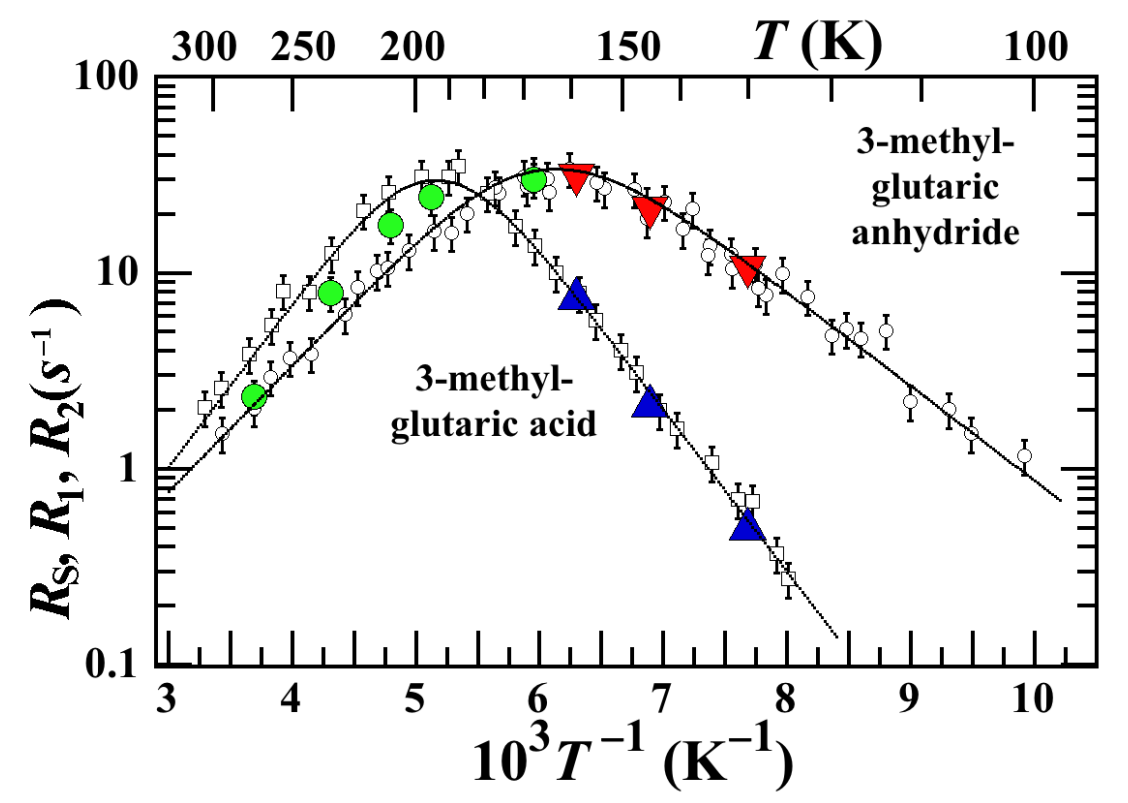

Fig. 7. The temperature $T$ dependence of the solid state ${ }^{1} \mathrm{H}$ spin-lattice initial relaxation rates $R_{\mathrm{S}}$ (O) and the rates of the two components $R_{1}(\boldsymbol{\nabla})$ and $R_{2}(\boldsymbol{\Delta})$ in a double exponential at $22.5 \mathrm{MHz}$ in sample $1 \mathrm{~B} 2$ (open to the air for one year) of 3-methylglutaric anhydride (1). The $R_{\mathrm{S}}(=R$ at lower temperatures) values of the pure samples of $\mathbf{1}(\bigcirc)$ and $\mathbf{2}(\square)$, along with the fitted solid line from Fig. 6, are shown for comparison. 


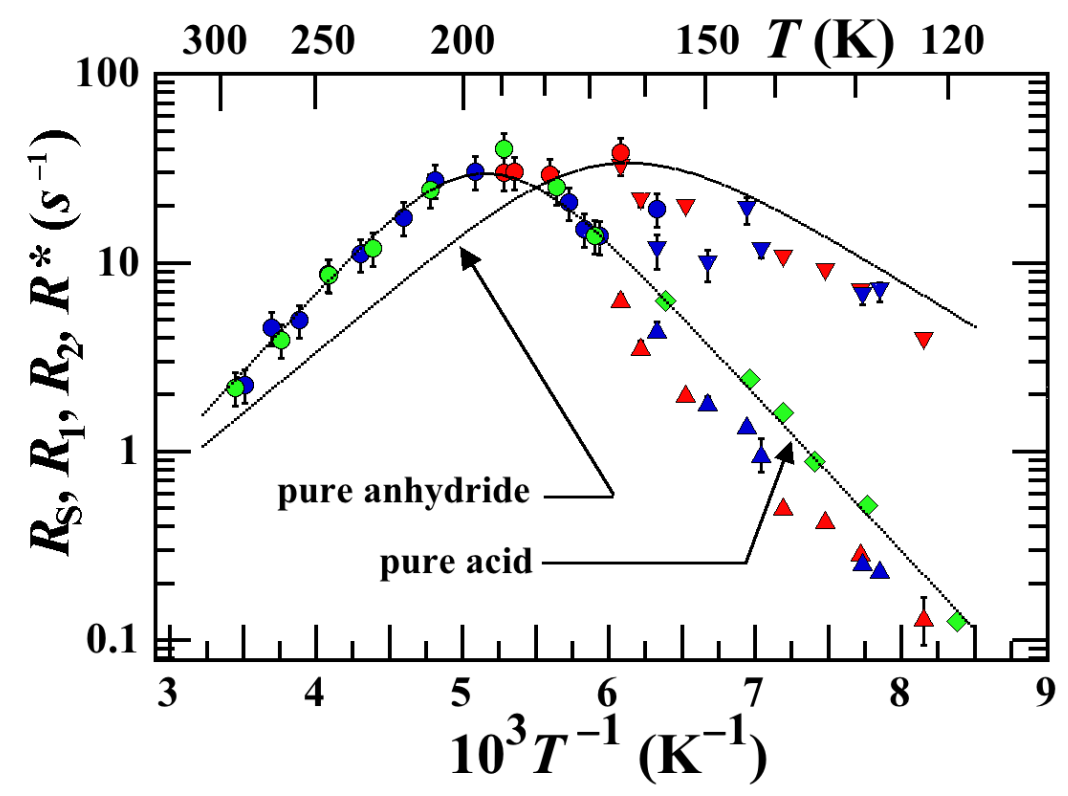

Fig. 8. The temperature $T$ dependence of the solid state ${ }^{1} \mathrm{H}$ spin-lattice initial relaxation rates $R_{\mathrm{S}}$ $(\bullet, \bullet, \odot)$ at higher temperatures, the double exponential relaxation rates $R_{1}(\boldsymbol{\nabla}, \boldsymbol{\nabla})$ and $R_{2}(\boldsymbol{\Delta}$, $\Delta)$ at lower temperatures, and the characteristic relaxation rates $R^{*}(\diamond)$ at lower temperatures, all at $22.5 \mathrm{MHz}$, in samples $\mathbf{1} \mathbf{A} \mathbf{1}(\mathbf{\bullet}, \boldsymbol{\nabla}, \mathbf{A}), \mathbf{1} \mathbf{A 2}(\boldsymbol{\bullet}, \boldsymbol{\nabla}, \mathbf{\Delta})$, and $\mathbf{1} \mathbf{A} \mathbf{3}(\ominus, \diamond)$. The lines, labeled by compound, are the fits to $R_{\mathrm{S}}$ (= $R$ at lower temperatures) versus $T^{-1}$ for the pure samples of 3methylglutaric anhydride (1) and 3-methylglutaric acid (2) shown in Fig. 6 and are repeated here for comparison. 


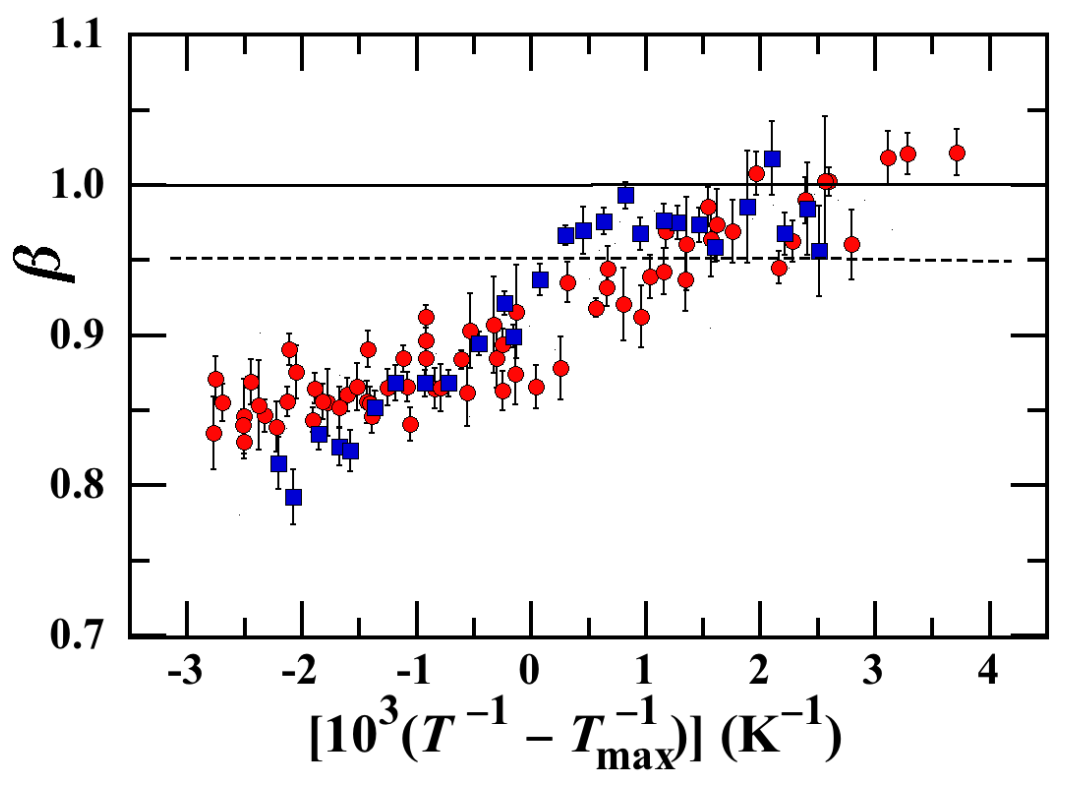

Fig. 9. The stretching parameter $\beta$ versus $T^{1}-T^{1}{ }_{\max }$ for temperature $T$ for several samples of 3methylglutaric anhydride (1) (O); sample 1B1 over the entire temperature range (103-290 K) and samples 1A1, 1A2, 1A3, and 1B2 (see Table 1) above 160K and samples 2B1, 2A1, and 2A2 of 3-methylglutaric acid (2) (ם) over the entire temperature range (130-300 K). $T_{\max }$ is the temperature of the relaxation rate maximum ( $160 \mathrm{~K}$ for $\mathbf{1}$ and $195 \mathrm{~K}$ for $\mathbf{2}$; see Fig. 6). The solid horizontal line indicates exponential relaxation $(\beta=1)$. Above approximately $\beta \sim 0.95$ (horizontal dashed line) it is difficult to distinguish, experimentally, on a run-by-run basis, between exponential and nonexponential relaxation. 